\title{
Collective rationality of group decisions in collaborative engineering
}

\author{
Stephen C-Y. Lu \\ The IMPACT Research Laboratory, \\ Viterbi School of Engineering, \\ University of Southern California, \\ Los Angeles, CA 90089, USA \\ E-mail: sclu@usc.edu
}

\begin{abstract}
Collaborative engineering is a dynamic socio-technical activity where a team of stakeholders works collaboratively to make group decisions based on collective rationality. This paper examines various impossibility conditions and possibility requirements for the existence of collective rationality from both theoretical and practical standpoints. Arrow's Impossibility Theorem is examined in light of the special characteristics of collaborative engineering problems. Since from a theoretical standpoint, no social welfare function can satisfy Arrow's rationality conditions of group decisions, this paper suggests some practical methods to guide collaborative engineering teams through teamwork and task-work iterations to approach collective rationality systematically when making group decisions.
\end{abstract}

Keywords: collective rationality; group decision; collaborative engineering.

Reference to this paper should be made as follows: Lu, S.C-Y. (2009) 'Collective rationality of group decisions in collaborative engineering', Int. J. Collaborative Engineering, Vol. 1, Nos. 1/2, pp.38-74.

Biographical notes: Stephen C-Y. Lu is permanent holder of the David Packard Chair in Manufacturing Engineering at University of Southern California (USC). He Heads the Master of Science in Product Development Engineering program at USC. He is the founding Director of the Improving Productivity via Advanced Collaboration Technology (IMPACT) research laboratory at USC. As a pioneer of the Engineering Collaboration via Negotiation (ECN) paradigm, he has published over 300 technical papers and served on many journals' editorial boards and keynote speaker in the fields of collaborative engineering. He is the Chief Editor of the International Journal of Collaborative Engineering. His current research interests are in design thinking, collaborative engineering, and technological innovation.

\section{Introduction}

As the complexity and diversity of engineering tasks increase rapidly, collaborative engineering has become a common practice in industry to make group decisions that are beyond the expertise or responsibility of individual engineers. The goal of collaborative engineering is to exploit the synergy between social teamwork and technical task-work to improve the quality and efficiency of group decision making by engineering 
teams (Lu et al., 2007a). In today's highly-connected global economy, companies must rely on the best practice of collaborative engineering to stay ahead of competitions when designing, producing, operating and maintaining complex products, processes and systems. Unfortunately, human collaboration in general and engineering collaboration in particular is more of a practiced art than a scientific discipline to date. We can often see the beneficial results from good engineering collaboration; however our abilities in understanding, conducting and teaching these desirable collaboration practices are very limited. A major barrier to the study of collaborative engineering and improvement of its practice is the lack of correct understanding of rationality, or more precisely the collective rationality, concept in group decisions (Lu, 2008). How to improve engineering collaboration to meet the growing social responsibilities and technical demands is the challenge faced by the entire engineering profession. This challenge calls for better understandings and rigorous treatments of how to attaining collective rationality in collaborative engineering teams.

Collective rationality has always been a highly contested subject within those research fields that are concerned with group decisions in various social sciences and engineering disciplines. Some examples in engineering include group design, system architecting, concurrent engineering, and collaborative engineering ( $\mathrm{Lu}, 2006)$. Many sharply contrasting viewpoints and heated debates can be found in a wide range of literatures. Some have based their arguments literally on historical results, such as Arrow's group-decision paradox from social choice research (Arrow, 1951) and von Neumann and Morgenstern's utility formulation in game theory (Neumann and Morgenstern, 1944), to insist on a rigid position in which collective rationality in group decisions is never possible. Hence, they argue that the only viable means for groups to make decisions is to place full trust in a dictator who can (and will) incorporate everyone's interests to make a rational decision unitarily. The implication of this belief is that, because collective rationality can never exist in theory, there are no intellectual merits or scientific values of study group decision in collaborative engineering practice. Meanwhile, other researchers have based their claims firmly on real-world evidences that, although the processes may not be most effective and the results are perhaps suboptimal, people one way or the other are always able to work out group decisions and reach joint agreements in practices (Scott and Antonsson, 2000). Therefore, they suggest that collective rationality must be somehow possible in real world; thus the above impossibility arguments are practically irrelevant and theoretically flawed - hence, they should be totally rejected. This position seems to imply that all is well with the intellectual bases of collective rationality; and therefore engineers can proceed to make group decisions without much hesitation or special caution. Both sides of this collective rationality argument keep accusing each other of failing to be theoretically rigorous or practically observant. As such, the debates of collective rationality continue, the progresses of group decision stall, and the practices of collaborative engineering suffer.

Although engineers have long been making group decisions in practice, the collective rationality debate has greatly intensified in the engineering research community recently. This is mainly due to the growing demands for more formal treatments of collaborative engineering that requires engineering teams to systematical make group decisions for increasingly complex assignments. Rather than choosing sides in, or rejecting the necessity of, the above collective rationality debate, we think that there are important reasons to rigorously study the scientific foundation of collective rationality of group 
decisions in collaborative engineering. However, we believe that those relevant theories and proofs from social sciences should not be taken literarily or applied hastily with only superficial consideration to make sweeping conclusions in engineering. Instead, we should thoroughly understand these social sciences knowledge (because collaborative engineering after all is a socio-technical activity) and carefully appraise them in light of the unique characteristics of engineering decisions, so that the theoretical requirements and practical limitations of collective rationality of group decisions in collaborative engineering can be better understood. Only through systematic investigations between engineering and social sciences knowledge can the collective rationality debates be settled and progresses made for collaborative engineering research. Unfortunately, such interdisciplinary investigations are very difficult for engineers who are unfamiliar with other non-technical disciplines, such as human cognition, macro economics, social choice, organisational behaviours and decision science, all of which are essential for the correct understanding of collective rationality in group decisions.

The purpose of this paper is to help engineers overcome the difficulties in understanding the notion of collective rationality in group decisions so that this concept can be properly employed in collaborative engineering research and practice. From the theory side, we carefully examine the rationality conditions specified in Arrow's theorem from social choice research to understand their theoretical limitations and practical implications with respect to the unique features of collaborative engineering practices. Our study concludes that, short of clean theoretical means to directly ensure the existence of collective rationality in group decisions via social welfare functions, other systematic group decision-making methods must be developed and employed to support engineering teams to make rational group decisions. As much as possible, the paper uses practical terminologies, concepts, and examples that are familiar by engineers to clarify the theoretical underpinnings and scientific results from social science researches. As such, some background materials presented in this paper may seem to be elementary for those readers with good social science knowledge. They are included here mainly for the benefit of engineering audiences, who are not familiar with these concepts based on their traditional engineering education. We hope that by using the plain language the paper can correctly depict collective rationality requirements, elucidate classical impossibility arguments, and explain some proven possibility conditions for engineers to benefit from social science results such as Arrow's theorem. As well, the paper recommends some helpful strategies and useful methods which collaborative engineering teams can use to systematically improve and finally approach collective rationality during group decision making.

\subsection{Our prescriptive collaborative engineering research strategy}

To date, the group decision problem has been studied from both empirical and theoretical angles. On the practical side, social psychologists and management researchers have proposed various empirical guidelines for group decision practice in teams. Although these 'descriptive' approaches are based on the best-practice benchmark, they cannot ensure the rationality of resulted group decisions. On the theoretical side, social choice researchers and decision theoreticians have developed various mathematical approaches to analyse and evaluate collective rationality of group decisions. These 'normative' formulations, although having a rigorous foundation to guarantee rationality, are hardly applicable in group decision practices due to their highly 
abstract modelling and idealised assumptions. As indicated in Figure 1, our collaborative engineering research has been focused on developing a theoretically-sound and practically-viable prescriptive approach between the two extremes of this spectrum.

Figure 1 Our prescriptive collaborative engineering research strategy (see online version for colours)

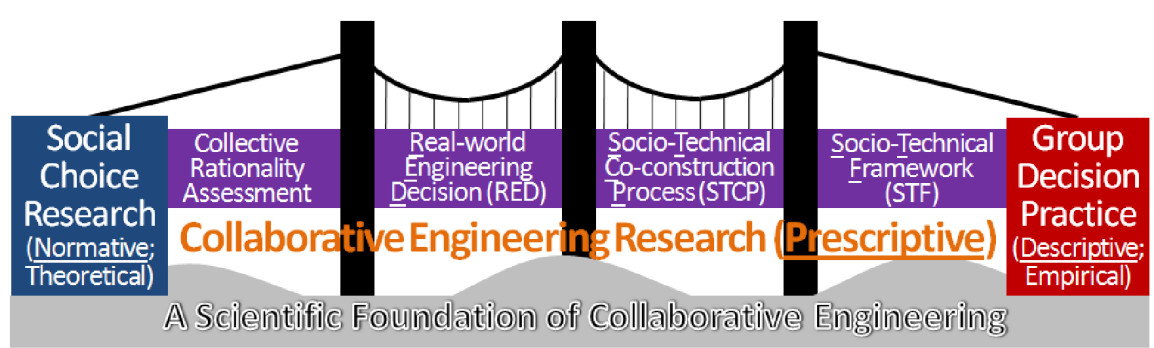

Our research goal is to establish a set of prescriptive guidelines, based on the rigorous theoretical foundation from social choice research and the practical considerations of collaborative engineering, which can direct group decision making toward collective rationality as much as possible. The key challenge is to strive for a good balance and maximise the synergy between the clean theoretical (or normative) and pure practical (or descriptive) approaches with sound scientific formulations and justifications. Based on systematic studies of collaborative engineering practices, we have established a scientific foundation of collaborative engineering ( $\mathrm{Lu}$ et al., 2007b) with this prescriptive orientation. This foundation sets the overall roadmap for collaborative engineering studies, of which one of the focal points is to develop principles and frameworks that can help engineering teams to administrate and approach collective rationality in group decision making practices. Staring from the practical end (see Figure 1), we have developed a Socio-Technical Framework (STF) for collaborative engineering ( $\mathrm{Lu}$ and Cai, 1999). The perspective model of the stakeholders, the process model of the tasks, and the conflict management model of the group decisions are the three key elements of STF. Then, a more detailed decision making process, called Socio-Technical Co-construction Process (STCP), is developed ( $\mathrm{Lu}$ and Cai, 2001), which extends STF to model social teamwork and technical task-work in collaborative engineering as a dynamic, co-construction process that creates team agreements via Participative Joint Decisions (PJD).

From the theoretical side (see Figure 1), we have investigated the foundations and limitations of the rationality conditions from Arrow's theorem, and suggested some practical strategies to approach collective rationality based on unique features of the collaborative engineering practice. The results of this study are presented in this paper. The practical usages of our research in STF, STCP and collective rationality for collaborative engineering are enabled by a Real-world Engineering (RED) decision framework (Lu, 2007). The RED framework organises collaborative engineering as a social teamwork phase that strives for collective rationality of the team and a technical task-work phase that seeks for global optimality of the assignment (Lu, 2007). As indicated in Figure 1, the combined capabilities from STF, STCP, RED and the results of collective rationality studies described in this paper completes our prescriptive 
collaborative engineering research that bridges the gaps between normative social choice research and descriptive group decision practice.

\section{Background and definitions}

In order to study collective rationality of group decisions in collaborative engineering with the right perspective, some background knowledge is necessary. This section presents the essential concepts and key definitions for readers to begin the correct understanding of this interdisciplinary subject. Section 2.1 explains what collaborative engineering is, and how it compares with the traditional engineering approach. Section 2.2 defines what a group decision is, and how it is different from an individual decision. Section 2.3 explains the collective rationality concept in group decisions, and clarifies its roles in multi-attribute decision-making problems in decision science research.

\subsection{What is collaborative engineering?}

Making real-world engineering decisions is never easy, because decision objectives and criteria are manifold, alternatives and consequences are unclear, and preferences and utilities are subjective. These difficulties greatly increase in collaborative engineering, where a team of engineers must jointly make group decisions to complete multi-faceted complex assignments. Because collaborative engineering involves social teamwork by multiple stakeholders and technical task-work amongst multiple objectives, the traditional reductionism philosophy from natural sciences and the classical optimisation framework from engineering analyses are inadequate to guide this practice. Section 2.1.1 presents a formal definition of collaborative engineering. Section 2.1.2 explains the differences between social and brute realities, both of which must be dealt with when making group decisions in collaborative engineering. Section 2.1.3 summarises the unique features of collaborative engineering practices, based on which some good group decision making strategies can be developed to improve collective rationality.

\subsubsection{A formal definition of collaborative engineering}

Collaborative engineering is a dynamic, socio-technical and team-based decision-making activity. It occurs whenever a team of stakeholders participates in a joint endeavour to make group decisions according to consensual agreements to accomplish complex assignments within (or across) the organisational boundary. Unfortunately, as an emerging practice, collaborative engineering has come to mean many things to many people. In order to study this subject correctly, we begin with a formal definition of collaborative engineering as:

"Collaborative engineering is a dynamic socio-technical group decision-making activity where an interdisciplinary team of stakeholders, who have shared values, different perspectives, and multiple preferences, work jointly to establish collective rationality in teamwork and then, based on which, to attain global optimality in task-work to accomplish an assignment with a common goal and limited resources." 
Stated differently, collaborative engineering is a human-centred activity that involves a dynamic socio-technical decision-making process to maximise the synergy between technical task-work by individuals and social teamwork by a collective (i.e., a team of stakeholders). Unlike traditional (e.g., natural) science-based engineering activities that are mainly concerned with individual technical decisions in task-work, collaborative engineering must additionally deal with group decisions and social interactions in teamwork. It is important to note that, in order to collaborate effectively, stakeholders must work together as 'teams' in an organisation, rather than acting as individuals or 'work groups' in a large open community. In organisational studies (Hellriegel et al., 1998), there is a major difference between what constitutes a 'team' and what a 'group' is. The main difference is that members in a team must share resources and outcomes (e.g., joint rewards or blames) to achieve a common goal via tight collaboration; whereas individuals in a working group may only share resources with others to improve their individual gains through loose coordination (Kozlowski and Bell, 2003). For example, engineers assigned to a new product development project must work closely as a 'team'; whereas students who study together in the library to prepare for the examination are more like a 'work group' (Lu, 2009).

The integration between teamwork and task-work is the hallmark of collaborative engineering. This important synergistic process produces a 'common understanding' of the given assignment among different participants, enabling them to 'do the right things' rationally as a collective in teamwork and then 'do the things right' optimally in task-work (Lu, 2007). Figure 2 shows an IDEF-0 (i.e., functional) description (IEEE, 1998) of the collaborative engineering process, which models teamwork and task-work as two different but interrelated 'functions' with inputs, controls/constraints, mechanisms, outputs, and feedbacks.

Figure 2 IDEF-0 functional description of teamwork and task-work in collaborative engineering (see online version for colours)

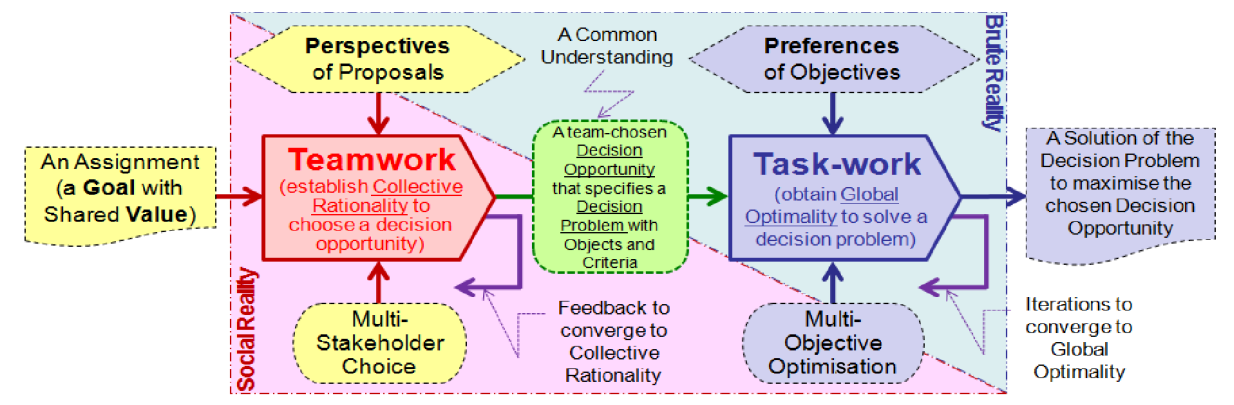

As shown in Figure 2, the input to the collaborative engineering process is an assignment given by an external party to the team. This assignment should come with a clearly identified 'goal' and some explicitly imposed corporate policies and/or implicitly assumed professional 'values' which team members are expected to follow. The collaborative engineering process starts with teamwork - a dynamic social interaction process whereby members (called stakeholders) first express their individual 'perspectives' toward various proposals (e.g., possibilities on how to approach the given assignment) to creatively explore and jointly choose a 'decision opportunity' that the team believes can best realise the set 'goal" ${ }^{\text {' }}$ of the given assignment. As will be 
explained in Section 2.2.1, 'perspective' is used here to represent the high-level preference of a decision body. Due to social interactions, stakeholders' initial perspectives will dynamically evolve (and hopefully converge to a common one, leading to the establishment of collective rationality) as teamwork progresses. The jointly-chosen decision opportunity in teamwork represents a 'common understanding' among tem members regarding how they believe the team can best tackle the given assignment. This decision opportunity, in turn, defines a specific decision problem consisting of a set of decision objectives and evaluation criteria (Keeney, 1992). Next, team members must solve this self-defined decision problem collaboratively during task-work using their different 'preferences' toward multiple alternatives and consequences. The final outcome from this two-stage collaborative engineering process is a group decision that represents the optimised solution to the decision problem (achieved in task-work) defined by the decision opportunity chosen by all members (done in teamwork) as a rational collective. In order words, members of the collaborative engineering team must first attain collective rationality in order to rationally choose a decision opportunity in teamwork before they can proceed to optimally solving a decision problem in task-work. In short, collective rationality ensures that the group decision made by the collaborative engineering team can result in the best possible solution to the decision problem that maximises the decision opportunity chosen by team members to achieve the set goal of a given assignment.

The hallmark of collaborative engineering is the team's ability to systematically establish collective rationality in teamwork, such that the 'two-heads-are-better-than-one' benefits can be fully realised (Blinder et al., 2000). Because group decisions involve dynamic social interactions that influence human preferences and in turn change consensual agreements, the decision mechanisms and rationality requirements in teamwork of collaborative engineering are different from that for task-work in traditional engineering (where decisions are more static and assumed to be made by individuals). For individual decisions, the classic rational decision theory (see Section 2.3.1), which presumes a self-interested decision body with full rationality and unlimited resources, can be used (see Section 2.3.2). In these classic cases, individuals' perspectives are static and decision preferences are assumed to be known, a priori; they are unaffected by social interactions taking place even when stakeholders work interactively in teams. Consequently, the rationality concept can be treated, more or less, statically and deterministically for individual decisions. In collaborative engineering, however, social interactions in teamwork always change individuals' decision perspectives, which, in turn, alter their decision preferences in task-work. For these highly dynamic cases, collective rationality is a much more complicated notion, and is best pursued via the constructionist approach (Restivo and Croissant, 2008) from social sciences (verse the deterministic approach from natural sciences). As will be made clear later, dynamic perspectives and evolving preferences of stakeholders occurred during social interactions have significant impacts on if, and how effectively, the team can achieve collective rationality in collaborative engineering.

\subsubsection{Social and brute realities in collaborative engineering}

Collaborative engineering is a socio-technical activity that uses social teamwork to accomplish technical task-work. Teamwork is a social interaction process where stakeholders work together to deliberate various social reality concerns of the given 
assignment (Hoegl and Gemuenden, 2001). Engineering task-work, on the other hand, is a technical problem-solving process where stakeholders employ brute reality knowledge to find solutions. Therefore, the notions of social reality in teamwork and brute reality in task-work (as indicated in Figure 1 by red and blue colours) are very important to the correct understanding of group decisions in collaborative engineering (Lu, 2009).

Brute reality encompasses deterministic knowledge, which is independent of human preferences. Brute reality knowledge is always absolute, objective, and observer-independent; they exist in Nature irrespective of human's interests - people cannot simply wish them away. Natural sciences use the determinism philosophy to discover brute reality knowledge which engineers must obey as absolute constraints when making technical decisions in task-work (e.g., to make sure that the designed product can function well in Nature). Social reality, on the other hand, consists of socially-constructed understandings based on human opinions and collective agreements influenced by social interactions (Collin, 2002). Comparatively speaking, social reality knowledge is relative, subjective, qualitative, and preference-driven than its brute reality counterpart. Social reality only remains valid when there are consensual agreements among concerned stakeholders. Engineers must understand social reality principles based on the constructionist approach (Holstein and Gubrium, 2007) to guide group decisions in teamwork (e.g., to make sure that the designed product is purposeful for the market). Since social reality concerns are always observer-dependent, they often lead to the so-called 'under-determined' outcomes (e.g., different teams of engineers work on the same product design assignment in teamwork can come up with complete different outcomes) (Lu, 2009). Under-determinism, which should not be confused with the 'chaotic' phenomena in natural systems, is very common in social reality systems and can be best understood by the constructionist thinking. Unfortunately, under-determinism and constructionist are often alien ideas to engineers who hold a strong deterministic philosophy from the traditional engineering paradigm.

The traditional (natural) science-based engineering paradigm focuses on understanding and applying brute reality knowledge deterministically to support the analyses of engineering decisions. In today's engineering practice, while engineers have many science-based engineering models to analyse decision consequences, optimise decision objectives, and solve decision problems in brute reality, the deliberation of human preferences to rationally choose decision opportunities based social reality concerns is often left out for other professions (e.g., marketing and management personnel). As a result, many well-functioned technical artifacts fail on the market because their functions optimised with brute reality cannot rationally satisfy customer preferences according to social reality (Simon, 1996). To stay competitive, engineers must learn how to use social reality principles to consider human preferences, in the same manner and with the same rigorousness, as natural science knowledge is used to deal with brute reality constraints. This is especially important when engineering decisions involve multiple stakeholders with multiple objectives, leading to a dynamic socio-technical endeavour as in the case of collaborative engineering.

\subsubsection{Some special features of collaboration engineering}

Collaborative engineering assignments often have high degrees of complexity, which call for diverse expertise beyond what any individuals can achieve alone. 
This is the chief reason for organisations to assemble engineering teams with shared responsibility and complementary knowledge to engage in collaborative engineering. Proper assembly of sufficient expertise among team members is critical for the success and effectiveness of collaborative engineering practice. If the combined expertise and aggregated experiences brought in by team members are sufficient for the given assignments, and if the team's social dynamics is such that all members are encouraged to fully participate in making group decisions jointly (i.e., Participative Joint Decisions (PJD) (Drenth and Koopman, 2008) with no dictatorship), the team should be able to systematically establish collective rationality to jointly choose the most promising decision opportunity and then effectively obtain global optimality to solve this decision problem. In this way, group decisions for complex assignments can be made promptly by the team without exhaustive searches or expensive iterations, as would be the case for individuals. Innovative new options for group decisions also become more possible because of the diverse expertise and multiple perspectives contributed by the members. This 'two-heads-are-better-than-one' feature of collaborative engineering (i.e., based on combined knowledge and aggregated experiences) has significant implication on the possibility requirements of collective rationality of group decisions for complex assignments as will be explained in Section 4.

Another distinguishing feature of collaborative engineering is the socio-technical nature of group decisions. In traditional engineering, decision making is mostly a technical activity by individuals where a decision opportunity (and its corresponding decision problem) is assigned by others. Engineers simply use brute reality knowledge of the problem domain to search for the optimal solution that maximises the given objectives (e.g., high performance, low cost, etc.). The decision objectives and evaluation criteria are treated as given conditions, leading to deterministic solutions to this externally assigned decision problem. Members of the collaborative engineering team, on the other hand, must first collaboratively choose the decision opportunity among themselves based on collective rationality established through social interactions during teamwork, before they can solve the self-identified decision problem to obtain global optimality during task-work. Since social interactions in teamwork are a dynamic process whose outcomes are non-deterministic (i.e., not predictable a priori), the final outcomes from collaborative engineering are always human-dependent. In other words, different teams and/or stakeholders work on the same collaborative engineering assignment can lead to different group decisions. Therefore, collaborative engineering is open-ended; its outcomes are not always deterministic as with the case of traditional engineering. Group decisions in collaborative engineering should be treated as a social construct (Section 2.1.2). As will be explained in Section 4, this social construction notion has a significant ramification on the meaning and existence of collective rationality of group decisions in collaborative engineering.

Besides the high-complexity and socio-technical features, collaborative engineering is still a technical activity where many quantitative empirical and analytical models of the domain can be used for its solutions. This technical nature puts collaborative engineering in a different category than many other kinds of human collective endeavours studied in social sciences. As will be explain later, one of the main factors that determine the possibility of collective rationality is the 'measurability' of individual preferences. If stakeholders' preferences are measurable, then their aggregations to establish collective rationality are possible (Keeney and Raiffa, 1993; Keeney, 1976). In general, qualitative measurements of preferences are harder to compare and aggregate than quantitative 
ones. For many social sciences activities (e.g., political election, for example) social reality is the main concern, domain knowledge is mostly qualitative, and human preferences are hard to quantify precisely. However, because of the technical nature of collaborative engineering task-work, many natural sciences domain models which are quantitative in nature with numerical representations can be used to evaluate and compare the relative merits of various alternatives. Some of these quantitative domain models can also be abstracted using various appropriation techniques (Osio and Amon, 1996) to develop qualitative surrogate models to support group decisions in teamwork at early stages of collaborative engineering. In short, due to the technical nature of engineering domain, quantitative measurements of possible consequences and stakeholder preferences are often possible in task-work (e.g., using detailed analytical models) and often probable in teamwork (e.g. using abstracted surrogate models). As will be explained later, this feature has a major impact on the existence of collective rationality in collaborative engineering.

Lastly, like any real-world engineering tasks, collaborative engineering assignments are often constrained by many practical limitations which restrict the possible types of decision alternatives and preference profiles that should/can be considered by the collaborative engineering team. For examples, organisation strategies and/or company policies can often eliminate some otherwise viable alternatives to be considered; government regulations can sometime dedicate the preference directions which engineers follow to explore possible alternatives (Scott and Antonsson, 2000). As well, market competitions and/or past experiences can often lead to restricted types of preference profiles (e.g., single-peaked preference) for some decision alternatives. In short, when practical considerations and/or physical limitations are introduced, decision options are never unlimited and preference types are seldom unconstrained for real-world collaborative engineering assignments. This feature of restricted domains and limited preference types also has a great consequence for the possibility conditions of collective rationality of group decisions in collaborative engineering, as will be explained later.

\subsection{What is group decision?}

Broadly speaking, a Decision $(D)$ can be a choice, a solution, a judgment, or an agreement resulting from some metal and cognitive processes by a decision body. A decision making activity $(\boldsymbol{\theta})$ can be specified by triplets of objective, alternative and preference (Bell et al., 1988). An Objective $(O)$ is a measurable milestone along the direction of attaining the goal of an assignment. An Alternative $(A)$ is a possible option whose outcomes can potentially satisfy the set objective. A Preference $(P)$ represents the decision body's aptitude to judge, trade-off, and select an alternative to its/their liking. In order to analyse a decision systematically, the objectives must be clarified by measurable Criteria $(C)$, the alternatives must be instantiated by possible consequences $(X)$, and the decision body's preferences must be articulated by meaningful Utilities $(U)$. Because $\boldsymbol{\boldsymbol { D }}\{O(C) ; A(X) ; P(U)\}=D$, a $(\boldsymbol{\theta})$ must carefully contemplate the specific forms and relationships of the $\{O(C) ; A(X) ; P(U)\}$ triplets.

A decision body can be either an individual or a collective. A group decision ${ }^{2}(\boldsymbol{G})$ is the result of $(\boldsymbol{D})$ when the decision body is a collective that consists of multiple stakeholders who make the decision jointly. Given an assignment by the external body, collaborative engineering teams make such group decisions collaboratively to achieve a common goal. 


\subsubsection{A preference structure of decision making}

Preference $(P)$, which represents human's cognitive ability to select something over others, plays a central role in $(\boldsymbol{D})$. Unfortunately, preference is highly subjective and driven by the decision body's different cultural, societal, educational and professional backgrounds. As such, human preference is always a social construct (or it is socially constructible) (Lichtenstein and Slovic, 2006) - i.e., it can be dynamically influenced by the decision body's social interactions with others. Furthermore, preference exists at multiple levels of detail and, therefore, can be expressed with varying degrees of specificity. This complication is especially apparent in group decisions; hence a more structural approach to preference is helpful. At a more abstract level, the decision body may just have some general inclinations or fuzzy viewpoints based on his or her broad backgrounds/experiences that can be used to qualitatively rank-order some preliminary ideas and/or initial proposals (e.g., potential directions of how to approach a given assignment). While at more detail stages, this general preference can develop into more specific ratings of the decision body's explicit preferences based on some quantifiable criteria toward the consequences of decision alternatives. According to social psychology studies (Lichtenstein and Slovic, 2006), the development, specification, and transformation of one's preference system in group decision making from abstract to detail, or vice verse, are also a 'socially constructible' dynamic process.

Our research adapts a 'Value-Perspective-Preference' hierarchical structure to represent the human preferential system from abstract to detail in group decisions. 'Value' ${ }^{3}$ represents the highest (i.e., most abstract) level of a decision body's preferential system, which is shaped by the long-term education, professional, cultural backgrounds and experiences (e.g., personal values). Additionally, there are also professional values that organisations would like (or require) engineers to follow when making decisions (e.g., corporate business ethics). These personal and professional values, both are social reality constructs, in turns, shape stakeholders' next-level liking in their preferential structure called 'perspective'. Perspectives refer to the stakeholder's general, but still comparatively qualitative, viewpoints (or attitudes) toward various decision opportunities being considered. Finally, the decision body's perspectives determine their 'preference' which represents the stakeholder's most detailed preferential system toward specific consequences of alternatives with respect to some quantifiable criteria of decision objectives of the decision problem.

This 'Value-Perspective-Preference' hierarchy of group decisions matches well with the teamwork and task-work collaborative engineering process described in Section 2.1.1

(see Figure 1). The collaborative engineering process is initiated when an assignment with a specific goal and a set of established professional 'Values' is given to the team. In teamwork, members first express their 'Perspectives' toward some preliminary proposals proposed by the team (e.g., strategies and approaches on how to best achieve the goal of the given assignment) to collectively choose (i.e., agree on) a specific decision opportunity to pursue. Aggregations of stakeholders' initial Perspectives, which are a social reality notion expressed as qualitative utilities (Section 2.2.2), lead to collective rationality which underlines the team's rational choice of the decision opportunity. This chosen decision opportunity from teamwork, in turn, defines a specific decision problem (with a set of agreed-upon decision objectives and evaluation criteria) (Keeney, 1992) to be solved by the team during task-work. Next, team members express and 
compare their individual 'Preferences' toward multiple alternatives to seek global optimality of the solution which maximises the set objectives with given criteria of the decision problem. Specific Preferences are treated as a brute reality concept expressed as quantitative utilities (see Section 2.2.2) to drive the optimal solution to this decision problem. In short, the decision body's high-level 'Values' drive their qualitative 'Perspectives' to rationally choose a decision opportunity in teamwork, which in turns establishes their quantitative 'Preferences' to optimally solve a decision problem in task-work. This progressive structure of Value (inherited in the assignment), Perspective (expressed during teamwork), and Preference (articulated during task-work) is very important to the proper understanding of collective rationality in group decisions in collaborative engineering.

\subsubsection{Represent decision preferences with ordinal and cardinal utilities}

A common way of using preference in decision making is to introduce the concept of utility ${ }^{4}$. In general, a Utility $(U)$ is defined as a measure of the expected 'true worth' of an outcome (e.g., relative satisfactions gained by obtaining or losing different consequences of alternatives). Economists have carefully distinguished between two kinds of utilities: cardinal utility and ordinal utility. Ordinal utility captures only qualitative ranking, but not the strength, of decision preferences (Debreu, 1954). On the other hand, cardinal utility additionally includes the quantitative magnitude of preference strength (Kenney, 1976). For example, one way of quantifying cardinal utility can be an alternative's probability of achieving an objective (Neumann and Morgenstern, 1953). Both utility kinds of formulation assign real numbers (e.g., utils) to consequence $(X)$ of Alternative $(A)$. For example, suppose an $A\left(X_{a}\right)$ has utility of 120 utils, $A\left(X_{b}\right)$ has a utility of 80 utils, and $A\left(X_{c}\right)$ has a utility of 40 utils. With ordinal utility, it would only be possible to say that $A\left(X_{a}\right)$ is preferred to $A\left(X_{b}\right)$ to $A\left(X_{c}\right)$, but no more. This is because ordinal utility treats the quantitative differences in utils as behaviourally meaningless - the ordinal utility values only encode an 'ordering' between alternatives, but nothing to quantify the relative strengths of preferences. However, with cardinal utility, it could be concluded that $A\left(X_{a}\right)$ is exactly the same amount better than $A\left(X_{b}\right)$ as $A\left(X_{b}\right)$ is better than $A\left(X_{c}\right)$. Because of the richer information content, more (e.g., interpersonal comparison and aggregation of preferences) can be done with cardinal utility than its ordinal counterpart.

The different representational powers of ordinal and cardinal utilities are significant to decision making in collaborative engineering, because the aggregation possibilities of human preferences are essential to the existence of collective rationality of group decisions. Depending on whether the decision is concerned with social reality in teamwork or brute reality in task-work, stakeholder's preference system, according to the "Value $\rightarrow$ Perspective $\rightarrow$ Preference" hierarchy as explained in Section 2.2.1, can be expressed by different kinds of utilities. In general, high-level Perspectives, which underline the rational choice of decision opportunities in social reality during teamwork, are more abstract and quantitative. They are socially constructible, and hard to compare and aggregate across multiple stakeholders. Hence, ordinal utility is the most practical (and oftentimes the only possible) way to represent the Perspective in social reality for group decisions during teamwork. On the other hand, detail-level Preferences, which derive the optimal solution of a decision problem in brute reality, are relatively specific and quantitative. Comparatively speaking, they are more measurable with 
quantifiable units, and hence are easily comparable and can be aggregated across multiple stakeholders. This is especially true for collaborative engineering due to the technical nature of its task-work (i.e., many quantitative domain models are available, as explain in Section 2.1.3). Therefore, cardinal utility is used in task-work to represent the Preference in brute reality when seeking global optimality for multiple objectives during task-work. In summary, we use ordinal utility to represent to the decision body's qualitative Perspectives (i.e., high-level preferences underline collective rationality) in social reality during teamwork, and cardinal utility to represent the decision body's quantitative Preferences (i.e., specific-level preferences underline global optimality) in brute reality during task-work.

\subsubsection{From individual decisions to group decisions}

With the above definitions, we are now able to explain the difference between individual and group decisions. When the decision body is an individual (or if all members act unitarily), the result of $(\boldsymbol{D})$ of is an individual decision $(D)$. When a decision is made by a collective that consists of multiple individuals (called stakeholders, $S$ ) with different perspectives, the result of $(\boldsymbol{\theta})$ is a group decision $(G)$. It should be noted that there exist different philosophical views toward the relationship between $(D)$ and $(G)$ in the research community. Some believe that all decisions are in fact individual decisions (Buchanan and Tullock, 1965); i.e., ( $(\boldsymbol{\theta})$ is never a real group activity. These people consider, in most cases, individuals make decisions independently as 'agents' for others (e.g., engineers make decisions for their teams or companies). Individuals must conform to the agreement they have made in accepting the responsibility to act as agent (Howard and Matheson, 1989). In other situations, the decision body may act as the 'principal' (e.g., the organisation to which engineers belongs) who devolves some decision making authorities upon an agent. The principal in this case is also making an individual decision in that devolution. Thus, whether as principal or agent, everyone is exercising individual decision making. This leaves no room for group decision making, always except that when individuals in the group must act collectively in accordance with, or in searching for, a single agreement.

Meanwhile, one could also take the view that no decision is ever a true individual decision in $(\boldsymbol{D})$. In the above cases, it may appear superficially so; but when we probe further we recognise how interrelated all decisions are with one another and any decision is the result of some group interactions (Keeney and Raiffa, 1993), with or without an explicitly assembled collective as the decision body. Certainly, a cyclic argument can be made that each group choice is ultimately determined by individual's decisions within the collective, and hence there are no true group decisions. In our opinion, attempting to resolve the philosophical arguments between $(D)$ and $(G)$ would not substantively contribute to the topic of our interest. Instead, we will focus the collective rationality discussion for those $(\boldsymbol{\theta})$ where multiple stakeholders, working in an explicitly assembled collective as a team (rather than just a work group), are actively participating in making a single Group decision $(G)$ jointly. This $(G)$ made by the entire collective ${ }^{5}$ is also called a team agreement in our research. Collective rationality must be established by the stakeholders themselves (as oppose to be imposed by a third party) in order to make a Group decision $(G)$ in collaborative engineering.

We should also point out that the classical decision analysis paradigm in decision science research is mainly suitable for supporting individual Decision $(D)$, whether 
the decision body is a principal or an agent (Kleindorfer et al., 1993). These analysis techniques can build an extra-personal decision model that can show the recommended alternative when various choices relevant to each stakeholder were used; and such analyses might be quite helpful in understanding the various positions of the parties involved. However, the Group decision $(G)$ cannot be understood directly using these classical decision analyses, in the sense that would be appropriate for an individual Decision $(D)$. Simply stated, the study of collective rationality in Group decision $(G)$ cannot be solely based on classical decision analysis techniques. Rather, we must seek additional knowledge from other related disciplines, such as social choice research, that directly address the aggregation of the preferences of the many to a consistent group preference of the few (i.e., the collective).

\subsection{What is collective rationality?}

Rationality is an important concept in decision making, especially when the decision body must collaboratively synthesise various social reality concerns to make a collective choice among multiple alternatives. As such, rationality has been studied extensively in many social science disciplines, such as philosophy, cognition, sociology, economics, and decision science, etc. Unfortunately, the rationality concept is often misunderstood by engineers, who are more familiar with the optimality concept often associated with brute reality knowledge. From both theoretical and practical viewpoints, rationality and optimality are two different notions ( $\mathrm{Lu}, 2008)$. The former is always subjective and is best approached with the constructionist thinking in social reality (Holstein and Gubrium, 2007); whereas the latter is more objective and can be investigated based on the reductionism philosophy in brute reality. In fact, we believe that several impossibility arguments insisted by some engineering researchers are the direct result of the confusion between collective rationality and global optimality. In this section, we well clearly define the concept of rationality (see Section 2.3.1), bounded rationality (see Section 2.3.2), and compare it with that of optimality (see Section 2.3.3).

\subsubsection{Rationality and rational decisions}

The word 'rational' is derived from the Latin word 'ratio' which means 'reason'. To be rational means having or exercising the ability to reason well in decision making. Hence 'rationality' generally refers to the quality (or state) of being rational in human's decision or action. Rationality is a hallmark of human cognition and rational decision making. It is characterised by the systematic reasoning ability of a decision body (individual or collective) to identify essential objectives to achieve the set goal, select proper criteria to characterise the objectives, estimate possible consequences to depict the alternatives, and articulate expected utilities to express the preferences. We should note that 'rationality' is a different, and broader, concept than 'logic' in scientific pursuits. The former includes 'uncertain but sensible' choices (or actions) based on probability, expectation, experience, and personal preferences; whereas the latter deals with provable facts and demonstrably valid relations between them (Power, 2004).

Rationality has different implications and requirements in the two reality regimes (see Section 2.1.2). In brute reality where knowledge is absolute, rationality can be treated as an objective notion that is closely linked to the concept of truth (i.e., proof-ability). In fact, if the world were only governed by pure brute reality, 
the proof-ability and rationality would become indistinguishable. In such an idealised 'black-and-white' world, a decision is either rational (i.e., true) or it is not (i.e., false) - there is no gradation, since there is no progression between valid and invalid knowledge according to natural sciences. However, as soon as social reality enters the world, rationality has more rooms for subjectivity by decision makers. Here, rationality refers to those sensible (and reasonable) human choices, agreements, or actions according to the Science of the Artificial (Simon, 1996). Rationality becomes a relative concept in social reality. As far as the social reality is concerned, all that is required for a decision body to be 'rational' is that he or she believes $X$, and that if $X$ then $Y$, so he or she chooses/does $Y$. Such a rational choice/action with respect to social reality is regardless of whether the logical relationship between $X$ and $Y$ is true (or false) in brute reality. In an extreme case where the world was completely governed by pure social reality without any brute reality consequences, the concepts of rationality and proof-ability would become totally distinguishable.

Based on the classic Rational Choice theory (Elster, 1986) that models decision making $(\boldsymbol{D})$ as a choose procedure (see Section 2.2.1), the 'rationality' of the decision body can be characterised by its aptitude to choose the most 'gainful' Alternative $(A)$ whose Consequences $(X)$ can yield the maxima utilities to meet the Criteria $(C)$ of a given Objective $(O)$. Unfortunately, with this classical formulation, the rationality notion which originated as a social science notion becomes somewhat intermingles with the optimality concept that has been studied extensively in engineering research as a brute reality idea. However, as will be made clear later that, especially when multiple stakeholders (i.e., $S>1$ ) making group decisions $(D)$ for multiple objectives (i.e., $O>1$ ), a globally optimised solution is not the collectively rational choice. Collective rationality is a human endeavour in social reality to derive choice functions and global optimality which is a technical activity in brute reality to seek optimised solutions must be treated differently in engineering decisions. Direct conversions between collective rationality and global optimality, although may be tempting in practice, are theoretically invalid. Correct understanding of the implications and requirements between the two notions in the contexts of social and brute realities is very important, because engineering decisions, especially group decisions in collaborative engineering, always involve both concepts at the cross-section between two different reality regimes.

\subsubsection{Bounded rationality and satisfying in decision making}

Collaborative engineering decisions must not only deal with brute and social realities, but also be guided by the practical limitation to rationality, called 'bounded rationality' which is a important concept developed from modern organisational behaviour research (Simon, 1956). In the classical rationality model, some rather unrealistic assumptions about the decision body and the world are made (Savage, 1954). They include:

- the decision body has precise information about exactly what will occur under any choice made; i.e., no uncertainties of consequences are considered

- the decision body has the unlimited cognitive ability to weigh every option against every other option; i.e., no constraints on resources are imposed

- the decision body is always aware of all possible options; i.e., no limits on information and knowledge are allowed. 
While many social science studies assume that humans can be reasonably approximated as 'rational' entities, most classical economics theories presume that people are always 'hyper-rational' (Young, 2001), and hence would never do anything to violate their preferences. However, real people, including engineers, are only 'partly rational' in their thinking, and sometimes are even emotional (or irrational) in parts of their decisions. In other words, in real-world decision making, there are always practical limits on the extents of human rationality. These limits are most apparent when decisions are made in the organisational settings, such as many collaborative engineering decisions which take place in corporate environments where resources (e.g., time, information, budget, etc.) are always limited.

We should understand that the bounded rationality concept does not reject the goal of trying to be rational (Simon, 1956); but it acknowledges the fact that, as much as people wants to be rational, there are always practical limits on how much rationality one can realistically achieve and afford in practice. Because of these realistic bounds on rationality, the decision body often lacks the cognitive capacities and/or practical resources to maximise (or optimise) their decisions in a strict manner in real-world decision making (Simon, 1956). But rather, the best they can do is to try to 'satisfice' (Simon, 1956), i.e., to select the first option that meets a given need or select the option that seems to address most needs rather than seeking the absolute 'optimal' solution that maximises all needs. Stated more formally, an alternative is optimal, if there exists a set of criteria that permits 'all' alternatives to be compared and the alternative in question is preferred by these criteria to all other alternatives (March and Simon, 1958). On the other hand, an alternative is satisfactory, if there exists a set of criteria that describes minimally satisfactory alternatives and the alternative in question meets or exceeds all these criteria (March and Simon, 1958). The 'satisficing' and 'bounded rationality' notions have significant implications in understanding the collective rationality concept in engineering decisions, which are always exercised with respect to limited resources, and an approximate (rather than exact) model of the actual situation. The elements of the situation are not given or static, but are themselves the outcome of dynamic sociological processes, including engineers' own decisions and the activities of others in the engineering team.

Unable to persistently seeking the absolute best via optimisation, engineers must try to strategically satisfy the absolute necessity instead in the most rational manner. This is true even when they face pure brute reality technical problems where complete knowledge is available (at least in theory) but practical resources, such as time and information, are limited. Organisational behaviour researchers have noticed that the satisficing approach occurs most often when a group of people looks towards a joint decision that everyone can agree on, even if it may not be the absolute best choice for every individuals in the group. We should note that in many real-world circumstances, a decision body might be totally uncertain about what really constitutes a satisfactory or a best outcome. If they choose the alternative which they 'believe' has the "maximum chance of being satisfactory", then this satisficing group decision will be in fact 'theoretically' indistinguishable from that of an optimising one (Bordley and LiCalzi, 2000). In this case, the rationality of a satisficing or an optimising decision becomes essentially isomorphic. But still, as will be made clear in Section 2.3.3, rationality for an optimised decision is conceptually different from that for a satisfied one. This difference is most significant when collective rationality is sought by multiple stakeholders to satisfy multiple objectives in collaborative engineering practice. 


\subsubsection{Rationality vs. optimality in engineering decisions}

Engineers must understand the difference between rationality and optimality in decision making to correctly deal with group decisions in collaborative engineering. Besides their dissimilar philosophical grounds, the practice difference between the two concepts is stemmed from the 'satisfying' and 'bounded rationality' notions discussed in Section 2.3.2. Decisions made with bounded rationality to satisfice the given objectives is certainly not 'optimal' in an absolute sense, but should be as 'rational' as possible (or practical) to satisfy the preferences or fulfill the purposes of the stakeholders. In an 'idealised' world where full rationality is possible, the disparity between rationality and optimality would blur. In the real-world, however, the bounded rationality and the satisfice concept bring their differences to the front burner in engineering decisions. Turning a blind eye on these differences is asking engineers to ignore all practical limitations of rationality that they must face in decision making, or demanding them to be always hyper-rational in their decisions. Neither is realistic in engineering practice. In many circumstances, a decision body might be totally uncertain about what actually constitutes an 'optimal' outcome due to the resource and/or information limitations resulted from bounded rationality. In these cases, if the decision body chooses the alternative which they rationally 'believe' has the "maximum chance of being the best satisfactory option", then this 'satisficing' choice made in practicality is conceptually the same as that of an 'optimising' one in their minds (Bordley and LiCalzi, 2000). Nevertheless, in the real world where bounded rationality always rules, rationality of an optimised solution in theory is different from that of a satisfied choice in practice. This difference cannot be ignored, especially when the decision opportunity must be agreed upon by a collective and the decision problem must be solved under multiple objectives.

To study collective rationality in group decisions properly, one must understand that optimality is a brute reality concern and rationality is a social reality issue. In our research, we define rationality as "making the most prudent choice to address social reality concerns" and optimality as "realising the best performance solution based on brute reality constraints". Referring to the Value-focused Thinking approach (Keeney, 1992), rationality underlines the rational choice of a decision opportunity and optimality drives the optimal solution of a decision problem. Rationality which strives to 'do the right thing', must precede optimality which attempts to 'do the thing right', - the former is the guidance to, and the anchor of, the latter in decision making (Lu, 2008). A decision body is ill-advised to optimise a decision problem before rationalising the choice of a decision opportunity that defines this decision problem. An optimised solution of an irrational choice is not useful in any case. Similarly, when multiple stakeholders face many objectives, such as group decisions in collaborative engineering, collective rationality in teamwork is the prerequisite of global optimality in task-work. In these situations, engineers can only optimise multiple objectives of the given assignment 'after' collective rationality among many stakeholders is established. Global optimisation without collective rationality is doomed to fail; trying to justify the rationality of a collective choice (e.g., a decision opportunity) after carrying out the optimisation of its solution (e.g., a decision problem) is risky. Therefore, although both collective rationality and global optimality play important roles in group decisions, the former must be understood thoroughly first (and foremost) in collaborative engineering research. 
Table 1 summarises and compares the different collaborative engineering, group decisions and collective rationality concepts discussed in Section 2, which establishes the necessary background for a systematic understanding of impossibility and possibility conditions of collective rationality.

Table 1 Key concepts of collective rationality of group decision in collaborative engineering

\begin{tabular}{ll}
\hline \multicolumn{2}{l}{ Collective rationality of group decision in collaborative engineering } \\
\hline (Social) Teamwork & (Technical) Task-work \\
(Subjective) Social reality & (Objective) Brute reality \\
Constructionist approach & Determinism approach \\
To 'Do the right thing' Rationally & To 'Do the thing right' Optimally \\
Collective rationality among multiple stakeholders & Global optimality among multiple objectives \\
Qualitative/Discrete option space & Quantitative/Continuous option space \\
Ordinal utility (Ranking) & Cardinal utility (Rating) \\
\hline
\end{tabular}

Common value $\rightarrow$ Qualitative perspectives $\rightarrow$ Quantitative preferences

\section{Impossibility of collective rationality in group decisions}

Rational individuals in a team must participate in group decisions based on collective rationality. The key question is if, and how, one can find some consistent ways to aggregate a set of private individual preference profiles into a single shared team preference so that a rational group decision can be made collaboratively by all members of the collective as a single whole. This section explains some classical theories and proven principles from existing social science research that address collective rationality (or the lack thereof) in group decisions. As an example from social choice research, which is an economics discipline that studies collective rationality, Section 3.1 presents the mathematical formation of collective rationality via a social welfare function. Arrow's Theorem is then explained in Section 3.2, which led to the conclusion of impossibility of rational group decisions under some specific collective rationality conditions, whose details are explained in Section 3.3. The famous group decision paradox derived from Arrow's seminal work is then presented in Section 3.4.

\subsection{An example mathematical formulation of collective rationality}

Collective rationality has been extensively studied in many subfields of social sciences that are concerned with Multi-Stakeholder Choice (MSC) problems. Different formulations and approaches exist. Some examples include microeconomics (e.g., social choice), sociology (e.g., social co-construction), and decision sciences (e.g., collaborative negotiation). In social choice research (Arrow, 1963), for example, a MSC problem is mathematically defined as:

$$
\begin{aligned}
& W=f\left(w_{1}, w_{2}, \ldots, w_{n}\right), \text { where } w \text { is 'welfare', and } \\
& \partial f / \partial w_{i}>0 \text { for all } i €\{1, \ldots, n\} \text { and } n \geq 2 .
\end{aligned}
$$


This formulation says that the Welfare $(W)$ of the collective is a function of the welfare (or interests, preferences) of all individuals belonging to this collective of $n$ members (note that the collective must have at least two members); and the collective welfare function that is good for the collective must result in increasing welfare for every member, indicating by positive derivative of the function (Gaertner, 2006). Since Welfare $(W)$ is a rather vague concept and is linked to human preference which is subjective, one can be more specific by representing (or quantifying) the Welfare $(W)$ as 'utility' (in the same manner as preference is quantified by utility, see Section 2.2.2). Then, the above welfare formulation can be restated as (Gaertner, 2006):

$$
\begin{aligned}
& U=k\left(u_{1}, u_{2}, \ldots, u_{n}\right), \text { where } u \text { is 'utility', and } \\
& \partial g / \partial u_{i}>0 \text { for all } i €\{1, \ldots, n\} \text { and } n \geq 2 .
\end{aligned}
$$

Certainly, both functions $f$ and $k$ can take many different mathematical forms; each specifies how the collective should behave in making group decisions (i.e., how to aggregate multiple individual preferences to form a single group preference). Some typical examples for the case of two stakeholders $(n=2)$ include:

$$
\begin{aligned}
\text { Linear additive: } U & =c_{1} u_{1}+c_{2} u_{2}, & & \text { where } c_{1}>0, \text { and } c_{2}>0 \\
\text { Exponents multiply: } U & =u_{1}^{\alpha} \cdot u_{2}^{\beta}, & & \text { where } \alpha>0, \text { and } \beta>0 \\
\text { A mixed form: } \quad U & =c_{1} u_{1}+c_{2} u_{2}+c_{3}\left(u_{1}^{\alpha} \cdot u_{2}^{\beta}\right), & & \text { where } c_{1}>0, c_{2}>0, \text { and } c_{3}>0 .
\end{aligned}
$$

Many other forms of aggregation function are also possible. A particular one that is of a special interest to rational group decisions in social choice research is called a "Social Welfare Function" (SWF) (Arrow, 1963). To be qualified as a SWF, its behaviours, properties and conditions (e.g., preference aggregation rules) must be precisely defined, so that collective rationality (or the lack thereof) of the group decisions resulted from the application of this SWF can be systematically predicted, evaluated and validated. Here, we use $\boldsymbol{R}$ to represent a specific SWF (i.e., a social preference order or an aggregation function) over 'all' conceivable social states (i.e., all available alternatives), $A=\{a, b, c, \ldots, m\}$ where $m \geq 3$ (i.e., more than three alternatives are available to the collective). Social choice scientists call this specific SWF $\boldsymbol{R}$ 'rational' (or having achieved collective rationality), if, and only if, it satisfies the following three basic properties (Arrow, 1963):

$1 \quad$ Reflexive $\rightarrow$ for all $a € \mathrm{~A}: a \boldsymbol{R} a$

2 Complete $\rightarrow$ for all $a, b € \mathrm{~A}$, and $a \neq b: \mathrm{a} \boldsymbol{R} b$ or $b \boldsymbol{R} a$

3 Transitive $\rightarrow$ for all $a, b, c € \mathrm{~A}:(a \boldsymbol{R} b$ and $b \boldsymbol{R} c)$ then $a \boldsymbol{R} c$.

The above reflexive and complete properties should be immediately obvious. However, the transitive property of $\boldsymbol{R}$ needs further comments. Unlike reflexivity and completeness, transitivity $\boldsymbol{R}$ can have different degrees (or extents), such as strict-transitive (defined above), quasi-transitive (Gibbard, 1973), and acyclical in decision-making practice (Gaertner, 2006). The latter two are formulated as follows:

Quasi-transitive $\rightarrow$ for all $a, b, c € \mathrm{~A}^{*}$ that is a subset of $\mathrm{A}:(a \mathrm{R} b$ and $b \mathrm{R} c)$ then $a \mathrm{R} c$; Acyclical $\rightarrow$ for all $a, b, c € \mathrm{~A}:(a \mathrm{R} b$ and $b \mathrm{R} c)$, then it is NOT the case that $c \mathrm{R} a$. 
All social choice research asks the same key question:

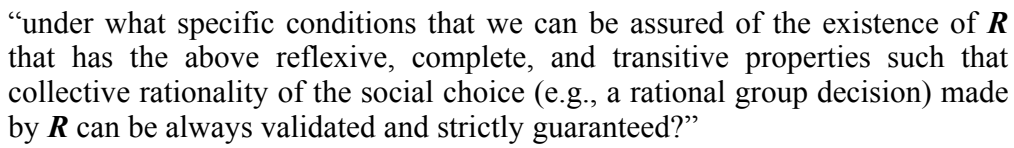

Stated differently, if $\boldsymbol{R}$, which has the reflexive, complete and transitive properties, can satisfy a particular set of rationality conditions then collective rationality will be guaranteed to exist in group decisions produced by this SWF $\boldsymbol{R}$. So, what should be the 'minimal' set of these rationality conditions, and how 'reasonable' are they in different practical applications? In fact, this question and its different answers have been at the centre place of the collective rationality debate in social choice research as will be explained next.

\subsection{The impossibility of collective rationality: Arrow's Theorem}

In 1950, Kenneth Arrow introduced an approach to analyse group decision making on social science issues that concern all members as a collective. The Arrow's Theorem concerns with the problem of aggregating multiple preferences of several individuals over a set of alternatives into a single common preference order over the same alternatives, such that this aggregated preference order can be seen as the group preference (or collective rationality) of the group as a whole (Arrow, 1951). This theorem has become the most well-known criteria for stating and contemplating the necessary, existence, and sufficient conditions of collective rationality in group decisions for many problems.

Formally stated, it is supposed that in a group with multiple stakeholders (hence, multiple preferences), a collective choice has to be made by the group from a set of alternatives $A_{i}$ (where $i=1, \ldots, n$, and $n>3$ ), and that each individual stakeholder $S_{j}$ (where $j=1, \ldots, m$, and $m>2$ ) of the group has a different preference order over these set of alternatives. Arrow asked a simple, yet very fundamental, question: how the group should decide what is the alternative that they judge 'best' as a collective? ${ }^{6}$ One way to answer this question is to search for a 'group decision function' (or a SWF $\boldsymbol{R}$ as defined in Section 3.1) that can map an ordered collection (or a profile $P$ ) of individual preference orders $\ddot{O}_{j}$ (where $\left.j=1, \ldots, v\right)$ over the set of alternatives $A_{i}$ (where $i=1, \ldots, n$ ) to a collective preference order $\ddot{O}$ over the same set of $A_{i}$. To be 'rational', this group decision function $\boldsymbol{R}$ should produce a reflexive, complete and transitive collective ranking of alternatives that allows for both strict preference $\left(A_{i}>A_{j}\right)$ and equivalence $\left(A_{i} \sim A_{j}\right)$ (Arrow, 1963). In other words, collective rationality calls for $\boldsymbol{R}$ to always map a profile of individual preference orders of the many (i.e., from multiple individuals) to a group preference order for the few (i.e., for the whole group), and the resulting group preference relation must be reflexive, complete and transitive in order to be called rational (or having achieved collective rationality).

\subsection{Specific collective rationality conditions of Arrow's Theorem}

The Arrow's Theorem (see Section 3.2) specifically considers the following requirements, constituting a set of reasonable conditions (herein called Arrow's 
Collective Rationality Conditions) of a fair (e.g., democratic and rational) group preferential ranking method for evaluating $\boldsymbol{R}$ (Arrow, 1951):

- Non-dictatorship (called Condition $D$ ): the SWF $\boldsymbol{R}$ should not simply follow the preference order of a special individual while ignoring all others.

- This collective rationality condition says that $\boldsymbol{R}$ must be sensitive to more than the wishes of a single stakeholder in the group. In other words, there is no single individual whose preference order 'alone' can determine the group preference order for the whole group (i.e., no dictatorship). This means that the following, for example, must NOT be true: "the group preference order, $A_{k}$ is preferred to $A_{1}$, if and only if a stakeholder $S_{1}$ prefers $A_{k}$ to $A_{1}$ ".

- Unrestricted domain or universality (called Condition $U$ ): the SWF $\boldsymbol{R}$ should create a 'deterministic' and 'complete' societal preference order from 'all-and-every' possible set of individual preference orders.

- In other words, $\boldsymbol{R}$ must lead to a result that can rank 'all' possible choices of alternatives relative to one another. $\boldsymbol{R}$ must be able to process all such possible sets of stakeholder preferences, and it should consistently give the same result for the same profile of stakeholders - no randomness is allowed in the application of $\boldsymbol{R}$. More precisely, it means that no profile of individual preference orders $\ddot{O}_{j}$ over any alternatives can be 'excluded' from the consideration. In essence, it requires that $\boldsymbol{R}$ must be able to deal with every combination of all alternatives as well as all types of preferences in the application domain.

- Independence of irrelevant-alternatives (called Condition I): this condition says that if we must restrict our attentions to a subset of alternatives and only apply the SWF $\boldsymbol{R}$ to those, then the result from this narrowed consideration should be compatible with the outcome when the whole complete set of options is considered.

- The Condition I means that any changes in individuals' rankings of irrelevant alternatives (i.e., ones that are outside the subset of current attention) should have no impact on the societal ranking of the relevant (i.e., currently considered) subset. In practice, this condition can be seen as a restriction on the sensitivity of $\boldsymbol{R}$. In other words, if $A_{k}$ is preferred to $A_{1}$ in a profile of individual preference orders over a set of alternatives and if an alternative (unequal to either $A_{k}$ or $A_{1}$ ) is removed from, or added to, the set of alternatives such that the individual preferences over the reduced, or expanded, option set don't change, then $A_{k}$ is again preferred to $A_{1}$ in the profile of the group preference order over this reduced (or extended) alternative set.

- Monotonicity or positive association of social and individual values (called Condition $M$ ): if an individual modifies his/her individual preference order by promoting a certain alternative over others, then the societal preference order should respond only by promoting that same alternative or at least not changing it (i.e., never by placing it lower than before). 
- This collective rationality condition simply says that an individual should not be able to hurt an alternative in group decisions by ranking it higher for himself or herself.

- $\quad$ Non-imposition or citizen sovereignty (called Condition $S$ ): every possible societal preference order should be 'achievable' by some set of individual preference orders. This means that the SWF $\boldsymbol{R}$ is mathematically 'surjective'.

- In mathematics, a function $F$ is said to be surjective (or 'onto') if its values span its entire co-domain; that is, for every $Y$ in the co-domain, there is at least one $X$ in the domain such that $F(X)=Y$. Said another way, a function $F: X \rightarrow Y$ is surjective, if and only if its range $F(X)$ is equal to its co-domain $Y$.

Another (and a somewhat 'stronger') version of Arrow's theorem was derived later (Arrow, 1963) by replacing the above Monotonicity and Sovereignty conditions together with a new condition as follow:

- $\quad$ Pareto efficiency (called Condition $\boldsymbol{P}$ ): if every individual stakeholder prefers a certain alternative to another, then so must the resulting group societal preference order.

- This collective rationality condition is, in fact, a demand that the SWF $\boldsymbol{R}$ will be minimally sensitive to the specific shapes (or particular properties) of the preference profile of individuals. In other words, if alternative $A_{k}$ is preferred to alternative $A_{1}$ in all individual preference orders $\ddot{O}_{j}$ in a profile $P$, then $A_{k}$ must be preferred to $A_{1}$ in the group social preference order $\ddot{O}$. Because this Pareto Efficiency condition is more concise and easier to understand than the combined effects of the above Monotonicity and Sovereignty requirements, we will use it instead of the conditions $\boldsymbol{M}$ and $\boldsymbol{S}$ in our remaining discussions.

In summary, Arrow concluded that:

"The above four rationality conditions together represented a minimal set of requirements for a social welfare function (SWF) to ensure the existence of collective rationality in group decisions with at least two stakeholders and minimally three alternatives."

Based on this conclusion, Arrow's General Possibility Theorem states that "there is no SWF that can satisfy the above four collective rationality conditions at once". Stated it differently, collective rationality cannot be guaranteed for group decisions derived from a SWF which fail to satisfy the above four conditions simultaneously. This statement is more commonly called Arrow's Impossibility Theorem (Arrow, 1963). We should note that Arrow, by no means implied that these four conditions exhausts the complete set of requirements that can reasonably be posed to evaluate collective rationality in group decisions (Franssen, 2005). He only proved that the list of these four conditions, if they are imposed 'together and at once', is already too long that, as soon as the number of alternatives to choose from is equal to or greater than three, it is impossible for a SWF between at least two stakeholders to find a $\boldsymbol{R}$ that can completely satisfy all the four collective rationality conditions. 


\subsection{The group decision paradox derived from Arrow's Impossibility Theorem}

Over the past half century, many have pondered the correctness and reasonableness of Arrow's above rationality conditions and his Impossibility Theorem. If these four rationality conditions are indeed reasonable and Arrow's derivation is theoretically correct, then, at least on the surface, this theorem seems to lead to a serious 'paradox' for group decision problems. The group-decision paradox says that it is impossible to algorithmically attain a SWF $\boldsymbol{R}$ in group decisions that involve at least two stakeholders with at least three alternatives (in other words, collective rationality of group decisions cannot be ensured by this $\boldsymbol{R}$ under these conditions). Some have taken Arrow's theoretical proof literarily to conclude that stakeholders can never decide together rationally when multiple alternatives are involved for problems with just some minimal levels of complexity. Such an inference, if remains unchallenged, would have a devastating consequence for group decision problems in application domains such as collaborative engineering.

We should note that there have been some disagreements in the engineering community (Scott and Antonsson, 2000; Franssen, 2005) regarding whether Arrow's paradox, which was motivated by social science problems, should be a concern at all in the engineering domain. At one extreme, some have taken an optimistic view to argue that due to the different natures of the domain, Arrow's Theorem does not apply in engineering decisions. Hence, the four social science collective rationality conditions that Arrow used have no direct bearing in engineering. At the other extreme, some researchers take a completely pessimistic view to insist on a very rigid position that Arrow's Theorem is unconditionally applicable to all problems; and there is no rational way out of this paradox in engineering. Consequently, they believe that dictatorship is still the most viable means to follow when engineers must make group decisions jointly in collaborative engineering.

To appreciate these opposing views, it is important to note that Arrow's original Theorem, and its resulting group-decision paradox, is only a neat mathematical proof of a theoretical group decision problem. Unfortunately, it has been loosely interpreted by many non-mathematical manners in practice, leading to erroneous statements, such as "no voting method (e.g., SWF) is fair", "every ranked method by groups is flawed", or "the only method that is not flawed is a dictatorship" (which rules out the merits of true group decisions). These hasty conclusions are often based on gross simplifications or misunderstanding of Arrow's original result and intent. In fact, as Arrow himself expressed hope at the end of his 1972 Nobel Prize lecture that (he wishes) others might take his result "as a challenge rather than as a discouraging barrier." This is exactly the challenge which collaborative engineering researchers must face. Stated differently, the engineering community should strive to seek the possibilities out of, and develop useful guidelines from, Arrow's Impossibility Theorem to support rational group decisions in engineering; rather than being blindly discouraged or constrained by this elegant theoretical result. Our research is aimed at meeting this challenge, and our results are explained in the rest of this paper. 


\section{Escaping the impossibility of collective rationality for group decisions in collaborative engineering}

After Arrow's seminal research, many scholars have attempted to escape his group-decision paradox by arguing that some of his collective rationality conditions are practically unreasonable and/or can be reasonably weakened in specific application domains (Sen, 1970). To understand these attempts, it is helpful to restate Arrow's rationality conditions with plain language. The Unrestricted Domain condition is to ensure the general applicability of the SWF; whereas the Pareto Efficiency and Non-dictatorship conditions are to guarantee the impartiality and faithfulness of the resulted group decisions (Franssen, 2005). These conditions seem to be intuitively reasonable' for most applications. But, the Independence of Irrelevant Alternatives condition (i.e., the group decision function can be informed only by the way the individuals order the alternatives with respect to each other) may not be immediately obvious to some. This requirement basically says that whether in the group preference order $A_{1}$ is preferred to $A_{2}$ (or $A_{2}$ is preferred to $A_{1}$, or whether they are judged of equal) must depend only on the pattern of individual relative preferences of $A_{1}$ compared to $A_{2}$, and not on how stakeholders order these two alternatives with respect to other (irrelevant) ones. Therefore, it also seems to be an adequate condition in many decision practices and application domains.

Can some of Arrow's rationality conditions be weakened or relaxed in the engineering domain for collective rationality to exist (or to be approached) in group decisions? To answer this question, we must exam the practical implications of Arrow's original work in light of the special features of collaborative engineering problems. This knowledge will enable us to determine if some of Arrow's rationality conditions can be reasonably altered that would not appreciably violate these basic rational principles in engineering teams. Furthermore, even though Arrow's theorem proved that no SWF $\boldsymbol{R}$ can satisfy the four rationality conditions simultaneously (hence it is impossible to derive a rational group decision directly from a social welfare function), it is still useful to understand these rationality conditions in collaborative engineering research, so that they can be treated as 'ideality', i.e., the most ideal state of affairs for teams to move closer to, when making group decisions,.

This section examines the unique features of collaborative engineering practice (see Section 2.1.3) to reveal the possibility of 'approaching' collective rationality in group decisions. The purpose is to be able to benefit from Arrow's research so that we can find some theoretical opportunities and/or practical means to guide engineering teams to approach collective rationality of group decisions in collaborative engineering practices (see Section 5). We should note that, the theoretical and practical extensions discussed in this Section, although fail to satisfy some of Arrow's rationality conditions, do not necessarily mean that they would lead to irrational engineering group decisions. They simply suggest that the particular circumstances for adapting these extensions must be carefully validated and justified, so that the engineering team can systematically move closer to the 'ideal' collective rationality of group decisions.

Section 4.1 discusses the measurability of human preferences and explains how engineers can take advantages of the measurable brute reality knowledge and surrogate domain models to quantify, compare and aggregate individual preferences to attempt collective rationality. Although the Pareto Efficiency Condition (i.e., collective preference order of the group must be consistent with that of the preference orders 
of individuals) is a generally sound requirement for teams that encourage participative joint decisions in collaborative engineering, this condition in Arrow's original Theorem is directly related to the full Transitivity requirement that is a rather restrictive provision hard to be completely fulfilled in engineering practice. Therefore, Section 4.2 uses the satisficing features of collaborative engineering (see Section 2.1.3) to weaken semi-Transitivity, hence alter Arrow's Pareto Efficiency Condition to attempt collective rationality. Then, Section 4.3 examines the issue relates to complementary domain expertise from multiple stakeholders to do away with the strict Non-dictatorship Condition in collaborative engineering teams. The Un-restricted Domain Condition in collaborative engineering group decisions is challenged in Section 4.4 based on the practical limitations of decision alternatives and allowable preferences in the engineering domain. Finally, the viability of the Independence of Irrelevant Alternatives Condition in collaborative engineering is discussed in Section 4.5 to reveal the possibility for approaching collective rationality.

\subsection{Measurability of preferences in collaborative engineering decisions}

Social choice research examines the rules for making group decisions based on the aggregation of individual preferences. Generally speaking, aggregation is only possible if individual preferences are measurable and Interpersonal Comparisons of Utility (ICU) is feasible. ${ }^{7}$ There exist fundamentally different views among social choice researchers regarding the measurability of human preference and decision utility. Pure 'Utilitarian' assumes that preference is measurable and comparable both individually and interpersonally (Stigler, 1968). In fact, most classical social choice research, including Arrow's Theorem, followed this pure utilitarian thinking. However, 'Non-Utilitarian' doubts that abstract human mental states, such as preference and utility, can be measured and compared at all (Shionoya, 1993). They criticised the strict ICU approach used in classical social choice research for falsely assuming that one could quantify the amount of satisfaction in group situations, and argued that group decisions based on comparing and aggregating individuals' utility gains and losses are never possible. While acknowledging the difficulty of measuring human mental states, many modern social choice researches (Hillinger, 2004) agree that 'limited' comparison of individual preferences is feasible for applications where stakeholders have some shared common backgrounds, cultures and experiences; or where the domains have some absolute knowledge (e.g., brute reality) that can be used for subjective evaluations. They generally accept the ICU approach in social choice research, as long as one is careful not to claim too much from the results. They suggest that, as much as possible, good group decisions should be based on decision variables which are not totally subject to malleable human mental states. In other words, as much as possible, ICUs should be based on real data (or real-valued variables) that are quantitatively measurable to make rational group decisions.

As stated in Section 2.2.2, ordinal utility is used for qualitative, and cardinal utility is good for quantitative, measures of human preferences. The ordinal utility theory states that, while the utility of a particular alternative cannot be qualitatively measured using an objective scale, a rational stakeholder, when deciding alone, should always be capable of rank-ordering different bundles of given alternatives quantitatively. However, inter-personal comparisons of multiple ordinal utilities from different stakeholders (and hence the relative preference strengths among team members) are not 
achievable by using such a qualitative manner. In fact, this is exactly the point that Arrow's impossibility theorem proved. In contrast, the cardinal utility theory suggests that decision utilities can be quantitatively measured and that the magnitude of this measurement is meaningful both individually and interpersonally (Barberá et al., 2004). As such, preferences, when expressed as cardinal utilities, can be quantitatively compared among multiple individuals and added up as a measure of the aggregated preference for these individuals. This means that cardinal utility allows for the measurability of preferences (and ICUs) across multiple stakeholders, making it possible to choose the greatest good of alternatives to the largest number of stakeholders in a collective, which is the ultimate goal of rational group decisions.

For many social science applications where data and knowledge are mostly qualitative, it is often impossible to quantify individual preferences as cardinal utilities. Fortunately, this difficulty is less severe in the engineering domain where many technical tasks have established quantitative models based on brute reality domain knowledge. As explained in Section 2.1.3, due to the quantitative nature of technical knowledge, utilities of decision alternatives are often quantitatively measurable in collaborative engineering. The measurability of individual preferences makes the comparison and aggregation of multiple preferences possible (Keeney and Raiffa, 1993), enabling engineers to escape the impossibility of collective rationality in group decisions. Mathematical simulations of these brute reality domain models can reveal much needed quantitative information that can be used to compare and rate different alternatives as cardinal utilities (rather than simply rank them with ordinal utilities).

Still, some may argue that, because of the general lack of detailed information for early decisions, cardinal utilities are often unavailable or unreliable at early stages (such as during the teamwork stage), and hence preference aggregations are still impractical. Fortunately, this is where the recent developments of "multi-layer surrogate modelling" approaches in large-scale simulation research become relevant and can be quite useful for collaborative engineering practice. Surrogate modelling (Queipo et al., 2005) typically begins with a large amount of quantitative data, collected from application cases or generated from computer simulations, of the domain. Given these organised databases, various multiple regression methods, such as statistics (Moses, 1986), machine learning (Witten and Frank, 2005), neural network (Gardner and Derrida, 1988), etc., are employed to automatically generate empirical 'layered models' with varying degrees of abstraction level of the quantitative domain knowledge. Abstract qualitative surrogate models, which are built directly from the more detailed quantitative domain knowledge, can be used to support early-stage discourses among stakeholders. It is possible to use these surrogate domain models to objectively rate, rather than just subjectively rank, initial proposals to establish collective rationality and jointly choose a good decision opportunity during the teamwork phase of collaborative engineering. Then, progressively more detailed domain models can be used to establish cardinal utilities at later stages, when stakeholders must quantitatively rate and compare multiple decision alternatives, during the task-work phase of collaborative engineering. In this way, cardinal utilities are available for rating (not ranking) early proposals and detailed alternatives at teamwork and task-work phases, leading to a rational group decision by collaborative engineering teams. 


\subsection{Satisficing and the semi-transitivity condition in collaborative engineering decisions}

Note that Arrow defined a SWF $\boldsymbol{R}$ as a 'complete' mapping from a profile of individual preferences to a collective preference profile over an 'entire' set of social options. He demanded this $\boldsymbol{R}$ 'must' have the ability to consistently rank 'all' options, so that it can be used to make a rational decision for the collective. Many researchers have asked whether such a highly restrictive requirement of completely ranking all options is reasonable or necessary in engineering practices. Based on the bounded rationality feature of the engineering domain (see Section 2.3.2), the answer to this question should be 'no'. For example, for many engineering tasks, not 'all' options are known, a priori, nor can be fully explored at once. Even in the very unlikely event when all options are available, it may still be too expensive and impractical to rank all of them to make a decision. Rather than "completely explore/compare all options" to optimise, engineers are often limited by available information, knowledge and resources such that the best they can do is to "carefully compare just a few most promising options" to satisfice the practical constraints. This satisficing approach is especially common in collaborative engineering teamwork, where a quick estimation of a few most promising options proposed by experiences team members based on some partial information at early stages is all what is needed and feasible.

However, a reasonable question may arise from this satisficing approach - how does the team know and be fully confident about what are the "most promising few options" without an exhaustive exploration and comparison? Interestingly, the answer to this question is exactly the main reason for engineers to engage in collaborative teamwork - i.e., to take advantage of the 'two-heads-are-better-than-one' (Blinder and Morgan, 2000) opportunity beyond what can be possibly achieved if they choose to work alone. Due to the complementary expertise and more information available from multiple team members (and assume that the team is well-organised with the necessary complementary expertise and good teaming practices), it can be reasonably expected that the team, as a whole, for the most time should be able to strategically identify a few promising options and quickly gather sufficient information than any individual can do it alone. In other words, although full rationality can only be guaranteed when all options are exhaustively explored and compared, this complete Transitivity requirement can be relaxed to some degrees when more domain expertise (e.g., heuristics and information) are brought in by multiple stakeholders in group decisions. In a well-assembled and highly experienced collaborative engineering team, if some members have proven track-records and rich experiences with some aspects of the domain and/or have successfully accomplish similar assignments before, the team can/should take advantage of this valuable expertise to quickly 'zoom in' the few most promising options. These collective heuristics enable the team to focus on 'the right direction' swiftly early stages without conducting exhaustive search and detailed comparison of all options to satisfy the full Transitivity requirement.

Furthermore, in Arrow's original Theorem the Pareto Efficiency Condition (see Section 3.3) is directly related to the full Transitivity requirement. If we accept the fact that a complete exploration and exhaustive comparison of all options is often impractical and unnecessary to make group decisions in collaborative engineering, then this full Transitivity requirement can be loosened from Arrow's original Theorem. Previous social choice research have already shown that, if the strict Transitivity 
requirement is weakened such that only the 'quasi-transitive' or 'acyclicity' property (see Section 3.1) is demanded, then some SWF $\boldsymbol{R}$ do exist which can completely satisfy Arrow's four conditions at once (i.e., collective rationality can exist) (Sen, 1969). Note that the strict Transitivity demands that if $A_{1}$ is preferred to $A_{2}$, and $A_{2}$ is preferred to $A_{3}$, then $A_{1}$ is preferred to $A_{3}$; whereas the 'acyclicity' property only asks if $A_{1}$ is preferred to $A_{2}$, and $A_{2}$ is preferred to $A_{3}$, then it is 'not the case' that $A_{3}$ is preferred to $A_{1}$. This is certainly a reasonable condition for group decisions in collaborative engineering practice, especially when the team has highly experienced members with complementary expertise (and is supported by a systematic group decision making framework ( $\mathrm{Lu}, 2007)$ ). In this case, collaborative engineering teams can use the satisficing approach to take advantages of collective heuristics in making rational group decisions.

The above practical remedy has a deeper theoretical implication. Previous social choice research has shown that if we remove the requirement that the Pareto Efficiency Condition must be strictly enforced across the 'entire and complete' set of alternatives, then some SWF $\boldsymbol{R}$ which completely satisfy Arrow's four conditions at once exist (i.e., collective rationality is possible). This has led to the so-called 'Pareto-extension' approach (Sen, 1969), which is commonly used in many social science applications to justify the existence of collective rationality. In collaborative engineering teamwork, this Pareto-extension concept gives us a theoretical base in that, as long as the alternatives under the current consideration (i.e., not the entire option set) comply with the Pareto Efficiency property, then the resulting group decisions can be regarded as 'rational'. Certainly, the degree for which the Transitivity property can be relaxed (or the extent to which the Pareto-extension condition is valid) depends largely on the richness of domain knowledge and the quality of the collective experiences that the collaborative engineering team possesses as a whole. This demonstrates the importance of good team assembly process and the need for a systematic decision support (Lu, 2007) in collaborative engineering practice. In short, if the team has the right assembly and mix of competent members who can collaborate effectively in exploring and comparing a few promising options and the team can follow a systematic decision making process through teamwork and task-work, then the satisficing approach and the semi-Transitivity requirement can lead to group decisions which are "rational enough" for all practical intents and purposes.

\subsection{Non-dictatorship condition in collaborative engineering decisions}

Non-dictatorship Condition means all votes from all members must count equally in group decisions. Such a principal is the foundation for a 'fair and democratic' system, when social reality is the main concern (e.g., in political election, etc.). On the surface, if we must insist on the collaborative, participative, transparent, and fairness requirements of all group decisions, then this condition also seems to be sensible in engineering. However, further examinations reveal that, unlike a democratic voting system in social sciences where everyone's opinion weights equally, engineering teams must take advantages of different opinions of stakeholders who have special technical expertise or unique professional responsibilities of some particular aspects of the assignment. This is because that engineering tasks are often knowledge-intensive - specific knowledge reserved (or demanded) for specialists often holds the key to the success. In fact, the opportunity of being swayed by some team members who know more about something 
than others is the very reason for engineers to work together in collaborative engineering teams. As well, since engineering teams always operate in some organisational settings, various implicit/explicit power distances and organisational structures (i.e., relevant social reality) will come into play when group decisions are being made by members with unequal social/organisational statures. In some situations, a few stakeholders may even be perceived (rightly or wrongly) by the team as having an implicit 'veto' power due to organisational or political implications. In other words, a pure and complete 'democratic' voting system never exists, and is not the norm in engineering practice; hence Arrow's Non-dictatorship Condition is not applicable in collaborative engineering.

In fact, previous social choice research have demonstrated that if this Non-dictatorship condition can be strategically weakened (i.e., modified but not completely removed), then a SWF $\boldsymbol{R}$ can still exist (i.e., collective rationality is attainable). Theoretical justifications and practical implications for the proposed modifications of the Non-dictatorship condition are available in many social choice literatures for different applications (Campbell and Kelly, 2007); they will not be repeated here. In the engineering domain, especially in light of the technical nature of domain knowledge and the bounded-rationality limitation, it is easy to understand the necessity for weakening this full democratic requirement in many collaborative engineering assignments.

\subsection{The unrestricted domain condition in collaborative engineering decisions}

To ensure the generality of SWF, Arrow's Unrestricted Domain Condition rejects any restrictions on the domain of considerations, in terms of the numbers (or scope) of alternatives that are to be explored and the types of preferences that can be expressed for these alternatives. In other words, Arrow says that a rational $\boldsymbol{R}$ can only exist in application domains that cover all different types of alternatives and preferences. The implication of this condition is that, if any restrictions on alternatives and preferences must be imposed, then the resulting group decisions for this restricted domain cannot be rational. This requirement presents another challenge for engineers to ponder, because engineering applications (including possible alternatives and acceptable preferences) are always constrained by brute reality knowledge - decisions for engineering problems can never be truly unrestricted. These 'hard constraints' cannot be ignored and must be completely followed when making engineering decisions. As well, besides complying with hard physical constraints, some market requirements from social reality (e.g., customer needs, competitor positions, etc.) must also be fully considered if the company wishes to remain competitive. These customers' needs and market competitions often dictate engineering decisions, and will restrict the scope of alternatives and the types of preferences that engineers can practically explore. Therefore, Arrow's Unrestricted Domain Condition is also often violated in engineering decisions. These violations seem to occur most frequently in large, complex engineering tasks when engineers are only able to pay attentions to the few most promising alternatives (instead of exhaustively exploring the 'complete' alternative set, see Section 4.2) due to the knowledge and resource constraints (i.e., the bounded reality concept, see Section 4.3) in organisations. Furthermore, there could be cases where some special considerations (such as reusing standard parts or previous designs) and other competitive reasons (such as beating a targeted market competition or meeting the sustainability requirements) would restrict 
the allowable types of alternatives and preferences, leading to decisions where Arrow's Unrestricted Domain Condition cannot be fulfilled.

Various strategies to modify the Unrestricted Domain Condition have been explored by social choice researchers for many social science problems (Black, 1958; Sen, 1966). For example, some research has shown that if there is only one criterion (or a single objective) by which the individual preferences are judged (i.e., example of a highly restricted domain), then all of Arrow's conditions can be met by the simple 'majority rule' voting method. ${ }^{8}$ Possibilities of rationality can also be found by using the so-called 'single-peaked preference' constraint (Arrow, 1963), which imposes some predetermined linear ordering of the alternative set in the domain of consideration. It has been proven that many SWF $\boldsymbol{R}$ can meet Arrow's rational conditions using voting mechanisms for these single-peaked domains (Arrow, 1963). This type of restricted single-peaked preference is commonly seen in engineering, because based on their unique technical expertise and professional responsibilities engineers often have a few particular options that they like the most along a preference direction. In other words, their dislike for an alternative would linearly grow larger as this particular alternative moves further away from that particular spot. This type of special preference is also quite common in engineering decisions where some common sense criteria are followed (e.g., the cheaper the better but not cheaper than the set cost, etc.). It has been proven that collective rationality can exist in such kinds of restricted domains with this special form of preference, which are very common in engineering practices.

\subsection{The independence of irrelevant alternatives condition in collaborative engineering decisions}

The failure of the Independence of Irrelevant Alternatives Condition is often demonstrated in social science literatures by the possibility of some 'cyclic preferences'. For example, if voters cast ballots as follows: Seven votes for $A_{1}>A_{2}>A_{3}$; six votes for $A_{2}>A_{3}>A_{1}$; and five votes for $A_{3}>A_{1}>A_{2}$. Then the collective preference of the group based on a simple ranked majority rule will be $A_{1}>A_{2}>A_{3}>A_{1}$ (i.e., a cyclic preference and an intransitivity occurs), which is deemed to be irrational according to the property of $\boldsymbol{R}$ defined in Section 3.1. Furthermore, consider that if a voting system currently picks $A_{1}$, and then $A_{2}$ suddenly drops out of the race, the remaining votes will then be: seven votes for $A_{1}>A_{3}$; and 11 votes for $A_{3}>A_{1}$. Thus, $A_{3}$ will win the election, even though the change (i.e., $A_{2}$ dropping out) concerned an 'irrelevant' alternative candidate who did not win in the original election. Various researchers have argued that this condition, if it is applied to the ranking of all alternatives, is an unreasonably strong requirement for a SWF $\boldsymbol{R}$ to exist. For example, some have suggested that as long as the 'intransitivity' of rankings does not appear among the top few most promising alternatives under current consideration (i.e., quasi-Transitivity property explained in Section 3.1 (Gibbard, 1973)), which is the common focus of engineering decisions, then everything regarding Arrow's paradox should be OK from the practical points of view. However, due to the fact that engineers can never be sure that the current few most promising alternative set will always remain as the top consideration when more information, time and resources become available at a later stage, this practical opportunity must be exercised very carefully in engineering decision.

We should also point out that this Independence of Irrelevant Alternatives Condition is typically grounded in the assumption that team members make group decisions with 
nothing stronger than a ranked preference order of the alternatives (i.e., the magnitudes and strengths of their individual preferences do not matter). In other words, ordinal, rather than cardinal, utility was used in Arrow's original formulation. This was because Arrow believed that, especially for the social choice problems which mainly concern with social reality, cardinal utility that requires detail quantifications and comparisons of stakeholders' individual preferences is impractical and unnecessary. Therefore, he only used ordinal utility, which is much easier to obtain with qualitative social reality knowledge, to represent the preferences in the development of his theorem. Researchers have already proven that if cardinal utility is used instead (i.e., stakeholders can compare the strengths of their individual preferences by rating alternatives (Keeney and Raiffa, 1993)), then a SWF $\boldsymbol{R}$ that satisfies all of the Arrow's conditions at once can indeed exist. As explained in Section 4.1, this is welcoming news for engineers because cardinal utility of alternatives, although is hard to obtain in other applications and early engineering teamwork (in social reality), can often be found in later engineering task-work using numerical simulation models based on continuous domain knowledge (in brute reality). The increased expressiveness of preference strengths is a unique feature of engineering tasks, which engineers must bear in mind when studying Arrow's group decision paradox.

\section{Summary: systematically approaching collective rationality of group decisions in collaborative engineering}

Rationality is the hallmark of any decision making, and collective rationality is the cornerstone of a sound group decision (see Section 2). Because preference underlines the rationality of individual decisions, different preferences from multiple individuals must be consistently aggregated when establishing collective rationality for the whole. As explained in Section 3, Arrow's group decision paradox is a clean mathematical proof of the non-existence of aggregation rules under a set of very particular rationality conditions for social science problems. However, these rationality conditions, although theoretically clean and conceptually rigorous, are not applicable for engineering problems where decisions must be based on both human preferences (i.e., social reality) and domain physics (i.e., brute reality). However, it is important to understand that Arrow's theorem, by no means, concludes that collective rationality is never possible for group decisions in engineering. Instead, it simply states that, under some particular conditions, no simple theoretical means, such as the use of social welfare functions as defined by Arrow, can exist that is able to fully ensure the rationality of group decisions in collaborative engineering. Short of the assurance by theory, collective rationality must be dealt with very carefully when making group decisions in collaborative engineering practice. This is one of the key challenges of collaborative engineering research.

The first step in meeting this challenge is to understand that, although Arrow's rationality conditions are pertinent to all multi-attribute decision problems in theory, because the essence of rationality differ greatly in social and brute realities, the degrees to which the rationality conditions can (and should) be imposed for engineering group decisions vary significantly. This understanding, in fact, is the 'point of departure' of our collaborative engineering research. Note that when optimality is pursued in brute reality, it can be treated as an absolute concept to be objectively evaluated and achieved. 
However, when rationality is applied to social reality problems, it becomes a relative notion which can only be subjectively expressed and approached. For group decisions that involve social reality, such as collaborative engineering teamwork where individual preferences are typically represented by ordinal utilities, the real or absolutely complete rationality would only exist in abstraction as 'ideality' (i.e., the most desirable state of group decisions to be attempted or moved closer to). Therefore, it is very important to understand that, when making collaborative engineering group decisions that involve both social and brute realities, the real challenge is not to prove or disprove the theoretical existence of collective rationality in some absolute terms, but rather to find sensible ways that, as much as possible, can help engineering teams to systematically approach the 'ideality' of group decisions. In fact, because of the relative and subjective nature of the rationality notion in social reality, it is not useful to treat collaborative engineering group decisions in a black-and-white manner (i.e., to judge them as either fully rational or totally irrational) when some of Arrow's rationality conditions are not fully satisfied. Instead, a more useful question to ask is, given that Arrow's conditions cannot be fully met, whether there could still be some means for engineers to make sound group decisions which are 'as rational as possible'.

The answer to the above question has been at the centre of our collaborative engineering research. Our research has proven that, while no simple and clean SWF $\boldsymbol{R}$ can be directly established and used to derive rational group decisions, rational group decisions can still be systematically approached by collaborative engineering teams by both practical and theoretical means. On the practical side, we have developed a Real-world Engineering Decision (RED) framework that structures collaborative engineering as integration and iteration between social teamwork and technical task-work ( $\mathrm{Lu}$, 2007). By modelling teamwork as a MSC problem and task-work as a Multi-Objective Optimization (MOO) problem, as well as by taking advantages of the structural similarity and cyclical dependency properties between MSC and MOO problems, the RED framework is able to guide collaborative engineering teams to systematically and gradually approach collective rationality in making group decisions. Details of the RED framework are presented elsewhere in $\mathrm{Lu}$ (2008) and will not be repeated here.

On the theoretical side, Section 4 in this paper has revealed that, at least, the following five opportunities exist:

1 Engineering task-works are of a technical nature, for which quantitative domain models based on brute reality knowledge are available for simulations, evaluations, comparisons, and rating of the relative merits of possible alternatives. As a result, preferences of technical decisions are more quantifiable than those of qualitative social science decisions. As explained in Section 4.1, social choice researchers have proven that, when both the order and relative strengths of individual preferences can be expressed (by cardinal utilities, for example), collective rationality can exist in group decisions. This presents a good opportunity for collaborative engineering teams to employ quantitative information of the technical domain, as much as possible, to attempt rational group decisions.

2 If collaborative engineering teams can be strategically assembled for members to bring in different know-how and complementary expertise, so that, as a collective, they possess a wealth of experiences which enables them to quickly explore a small set of most promising alternatives heuristically. In fact, with a well-assembly team, 
engineers often can rapidly establish the most relevant 'bottom lines' and then swiftly identify a few good alternatives that satisfice these important requirements as group solutions. As explained in Section 4.2, social choice research have shown that, if the full-transitivity requirement is altered so that only 'quasi-transitive' or 'acyclicity' is necessary, then some SWFs which satisfy Arrow's four conditions at once can exist (i.e., collective rationality exists). This suggests that well experienced engineering teams can use the heuristic-based satisfcing approach to effectively attempt rational group decisions in collaborative engineering practices.

3 Unlike democratic voting where each individual voice should count equally, the value of the different expertise among collaborative engineering team members must be strategically recognised and exploited. Giving more weights to experts' opinions in making group decisions does not mean that dictatorship is encouraged in collaborative engineering teams. Rather, as long as the principle of participative joint decision is exercised, it is simply a smart thing to do for the team to benefit from the collective wisdoms in teamwork. As explained in Section 4.3, social choice research have proven that if the non-dictatorship condition can be strategically weakened (i.e., modified but not completely removed), then a SWF $\boldsymbol{R}$ may exist (i.e., collective rationality is attainable) for group decisions in the domain. This implies that, with prudent justifications, engineering teams can still approach collective rationality in group decisions, while taking advantages of expert knowledge from individual members.

4 Engineering decisions are never truly unbounded in practice. Many restrictions on allowable alternatives or possible preferences exist in engineering due to hard constraints by brute reality knowledge, corporate policies, market competitions, etc. Therefore, engineers always make decisions in restricted domains, which is different from the problems considered by Arrow in his theorem. While not universally applicable, some types of domain restrictions, such as single evaluation criterion and single-peaked preference, are quite common in engineering practice. As explained in Section 4.4, social choice researchers have proven that SWF $\boldsymbol{R}$ can satisfy Arrow's rational conditions using simple voting mechanisms for single-peaked domains. This indicates that, at least for some types of engineering problems, collective rationality of group decisions can be attempted even under domain restrictions.

5 Similar to the above justifications for using the satisficing approach and relaxing the semi-transitivity condition, same arguments can be made for collective engineering teams to take advantages of their collective experiences to deal with the requirement of Independence of Irrelevant Alternatives in Arrow's original theorem. As explained in Section 4.5, as long as the intransitivity difficulty of ranking alternatives does not appear among the top few most promising alternatives under current consideration, then problems regarding Arrow's group decision paradox should be tolerable from the practical points of view. As well, researchers have proven that if cardinal, instead of ordinal, utility is used (i.e., stakeholders can compare the strengths of their individual preferences by rating alternatives), then a SWF $\boldsymbol{R}$ that satisfies all of the Arrow's conditions at once can indeed exist. This suggests that, even when the Independence of Irrelevant Alternatives condition is not fully followed, rational group decisions are still possible by collaborative 
engineering teams as long as different individual preferences can be quantified with brute reality knowledge.

In summary, we can conclude that the core challenge of collaborative engineering research is to guide engineering teams to strive for collective rationality in group decisions, which requires a balanced prescriptive integration between the descriptive studies of group decision practices and the normative investigations of social choice research. While Arrow has proven that collective rationality cannot exist for group decisions under a specific set of conditions, our research has established that there are still viable opportunities for collective engineering teams to attempt rational group decisions. This paper has demonstrated that, short of theoretical means to systematic aggregate individual preferences via social welfare functions to form a preference for the collective directly, the team can strategically take advantages of the unique characteristics of collaborative engineering problems to systematically exploit the possibility of attempting collective rationality in group decisions. We believe that, rather than regarding Arrow's theorem as an impossibility proof of rational group decisions as has been done historically, engineering researchers should, in fact, study it as a sound theoretical basis to explore the possibility of collective rationality of group decisions in collaborative engineering.

\section{References}

Arrow, K.J. (1951) Social Choice and Individual Values, 1st ed., J. Wiley, New York, NY.

Arrow, K.J. (1963) Social Choice and Individual Values, 2nd ed., Published by Yale University Press, New Haven and London, ISBN 0300013647, 9780300013641.

Barberá, S., Hammond, P.J. and Seidl, C. (2004) Handbook of Utility Theory, published by Springer, ISBN 1402077149, 9781402077142.

Bell, D.E., Raïffa, H. and Tversky, A. (1988) Decision Making: Descriptive, Normative, and Prescriptive Interactions, published by Cambridge University Press, ISBN 0521368510, 9780521368513.

Black, D. (1958) The Theory of Committees and Elections, published by Cambridge University Press.

Blinder et al. (2000)

Blinder, A.S. and Morgan, J. (2000) Are Two Heads Better Than One?: An Experimental Analysis of Group vs. Individual Decisionmaking, National Bureau of Economic Research NBER Working Paper No. W7909, Available at SSRN: http://ssrn.com/abstract=242143

Bordley, R. and LiCalzi, M. (2000) Decision Analysis Using Targets Instead of Utility Functions, published by Springer Milan, ISBN: 1593-8883 (Print) 1129-6569 (Online).

Buchanan, J.M. and Tullock, G. (1965) The Calculus of Consent: Logical Foundations of Constitutional Democracy, published by University of Michigan Press, ISBN 0472061003 , 9780472061006.

Campbell, D. and Kelly, J. (2007) 'Social welfare functions that satisfy Pareto, anonymity, and neutrality, but not independence of irrelevant alternatives', Social Choice and Welfare, Springer, Vol. 29, No. 1, July, pp.69-82.

Collin, F.S. (2002) Social Reality, Published by CRC Press, ISBN $0203047923,9780203047927$.

Debreu, G. (1954) Representation of a Preference Ordering by a Numerical Function, Cowles Foundation Paper 97, Reprinted from Thrall, Davis and Coombs, eds., Decision Processes, John Wiley, Hoboken, NJ. 
Drenth, P.J.D. and Koopman, P.L. (2008) 'Participative decision making: a comparative study', Industry Relationships: A Journal of Economy and Society, Vol. 18, No. 3, June, pp.295-309.

Elster, J. (Ed.) (1986) Rational Choice, Basil Blackwell, Oxford.

Franssen, M. (2005) 'Arrow's theorem, multi-criteria decision problems and multi-attribute preferences in engineering design', Research in Engineering Design, Vol. 16, Nos. 1-2, pp.42-56.

Gaertner, W. (2006) A Primer in Social Choice Theory, Published by Oxford University Press, London, ISBN $0199297509,9780199297504$.

Gardner, E.J. and Derrida, B. (1988) 'Optimal storage properties of neural network models', Journal of Physics A, Vol. 21, pp.271-284, doi:10.1088/0305-4470/21/1/031.

Gibbard, A. (1973) 'Manipulation of voting schemes: a general result', Econometrica, Vol. 41, pp.587-601.

Hellriegel, D., Slocum, J.W. and Woodman, R.W. (1998) Organisational Behavior, 8th ed., Southern-Western College Publishing, Cincinnati, Ohio.

Hillinger, C. (2004) On the Possibility of Democracy and Rational Collective Choice, Discussion Paper 2004-21, University of Munich, http://ssrn.com/abstract=608821.

Hoegl, M. and Gemuenden, H.G. (2001) 'Teamwork quality and the success of innovative projects: a theoretical concept and empirical evidence, organization science', Vol. 12, No. 4, July-August, Published by: INFORMS, pp.435-449.

Holstein, J.A. and Gubrium, J.F. (2007) Handbook of Constructionist Research, Published by Guilford Press, ISBN 159385305X, 9781593853051.

Howard, R.A. and Matheson, J.E. (1989) Readings on the Principles and Applications of Decision Analysis, Published by Strategic Decisions Group, London, ISBN 0962307408, 9780962307409.

IEEE (1998) IEEE Standard for Conceptual Modeling Language Syntax and Semantics for IDEF1X, IEEE Std., 13-20 February, New York, p.iii.

Jing, N. (2009) Utilizing Structured Arguments for Collaborative Negotiation of Group Decision in Software Design, PhD thesis, Department of Computer Science, University of Southern California, March.

Keeney, R.L. (1976) A Group Preference Axiomatization with Cardinal Utility, Management Science, International Institute for Applied Systems Analysis, Laxenburg, Austria.

Keeney, R.L. (1992) Value-Focused Thinking: A Path to Creative Decision-Making, Harvard University Press, Cambridge.

Keeney, R.L. and Raiffa, H. (1993) Decisions with Multiple Objectives-Preference and Value Tradeoffs, Published by Cambridge University Press, London, ISBN: 0521438837.

Kleindorfer, P.R., Kunreuther, H. and Schoemaker, J.H. (1993) Decision Sciences: An Integrative Perspective, published by Cambridge University Press, ISBN $0521338123,9780521338127$.

Kozlowski, S.W.J. and Bell, B.S. (2003) 'Work groups and teams in organizations', in Borman, W.C., Ilgen, D.R. and Klimoski, R.J. (Eds.): Comprehensive Handbook of Psychology (Vol. 12): Industrial and Organizational Psychology, Wiley, New York, pp.333-375.

Lichtenstein, S. and Slovic, P. (2006) The Construction of Preference, Published by Cambridge University Press, London, ISBN 0521834287, 9780521834285.

Lu, S.C-Y. (2006) 'Beyond concurrent engineering: a new foundation for collaborative engineering', International Journal of Advanced Manufacturing Systems, Vol. 9, No. 2, pp. $27-40$.

Lu, S.C-Y. (2007) Optimality and Rationality in Design Thinking, Working Paper.

Lu, S.C-Y. (2008) Advanced Mechanical Design - Lecture, University of Southern California, Los Angeles, CA 90007.

Lu, S.C-Y. (2009) Collaborative Engineering Principle and Practice - Lecture, University of Sothern California, Los Angeles, CA 90007. 
Lu, S.C-Y. and Cai, J. (1999) 'Modelling collaborative design process with a socio-technical framework', Proc. Sixth ISPE Int'l Conf. Concurrent Engineering, Bath, UK, pp.54-62.

Lu, S.C-Y. and Cai, J. (2001) 'A collaborative design process model in the socio-technical engineering design framework', Artificial Intelligence for Engineering Design, Analysis and Manufacturing, Vol. 15, pp.3-20.

Lu, S.C-Y., Elmaraghy, W., Schuh, G. and Wilhelm, R. (2007a) 'A scientific foundation of collaborative engineering', Annals of CIRP, No. 2, p.52.

Lu, S.C-Y., Elmaraghy, W., Schuh, G. and Wilhelm, R. (2007b) 'A scientific foundation of collaborative engineering', CIRP Annals - Manufacturing Technology, Vol. 56, No. 2, pp.605-634.

March, J.G. and Simon, H.A. (1958) Organizations, Published by Wiley, Hoboken, NJ.

Moses, L.E. (1986) Think and Explain with Statistics, Addison-Wesley, Reading, MA, pp.1-3.

Neumann, V.J. and Morgenstern, O. (1944)

Neumann, V.J. and Morgenstern, O. (1953) Theory of Games and Economic Behavior, Princeton University Press, Princeton.

Osio, I. and Amon, C. (1996) 'An engineering design methodology with multistage bayesian surrogates and optimal sampling', Res. Eng. Des., Vol. 8, No. 4, pp.189-206.

Power, D.J. (2004) Decision Support Systems: Frequently Asked Questions, published by iUniverse, ISBN $0595339719,9780595339716$.

Queipo, N.V., Haftka, R.T., Shyy, W., Goel, T., Vaidyanathan, R. and Tucker, P.K. (2005) 'Surrogate-based analysis and optimisation', Progress in Aerospace Sciences, Vol. 41, pp.1-28.

Restivo, S. and Croissant, J. (2008) 'Social constructionism in science and technology studies', in Holstein, J.A. and Gubrium, J.F. (Eds.): Handbook of Constructionist Research, Guilford, NY, pp.213-229, ISBN 9781593853051.

Savage, L.J. (1954) The Foundations of Statistics, Published by Wiley, Hoboken, NJ.

Scott, M.J. and Antonsson, E.K. (2000) Arrow's theorem and engineering design decision making', Research in Engineering Design, Vol. 11, No. 4, pp.218-228.

Sen, A.K. (1966) 'A possibility theorem on majority decisions', Econometrica, Vol. 34, pp.491-499.

Sen, A.K. (1969) 'Quasi-transitivity, rational choice and collective decisions', Review of Economic Studies, RePEc:bla:restud, Vol. 36, No. 107, pp.381-393.

Sen, A.K. (1970) Collective Choice and Social Welfare, Published by Holden-Day, San Francisco, ISBN 0816277656, 9780816277650.

Shionoya, Y. (1993) 'A non-utilitarian interpretation of pigou's welfare economics', in Koslowski, P. (Ed.): The Good and the Economical, Springer-Verlag, Berlin.

Simon, H.A. (1956) 'Rational choice and the structure of environments', Psych. Rev., Vol. 63, pp.129-138.

Simon, H.A. (1996) The Sciences of the Artificial, 3rd ed., MIT Press, ISBN 0262691914, 9780262691918, Cambridge, MA.

Stigler, G.J. (1968) 'The development of utility theory', Utility Theory: A Book of Readings, John Wiley \& Sons, New York.

Witten, I.H. and Frank, E. (2005) Data Mining: Practical Machine Learning Tools and Techniques, Morgan Kaufmann, ISBN: 0-12-088407-0.

Young, H.P. (2001) Individual Strategy and Social Structure: An Evolutionary Theory of Institutions, published by Princeton University Press, ISBN 0691086877, 9780691086873. 


\section{Notes}

${ }^{1} \mathrm{We}$ should point out that 'goal' is a different concept from 'objective' in decision science research. A 'goal' represents a general direction toward an increasingly more desirable state of affairs; whereas an 'objective' is a specific and measurable milestone along this desirable direction with a target time for completion. For example, one can set a 'goal' to become rich with a specific 'objective' of being a millionaire by the end of 2010. In collaborative engineering, goal is often set by an external party whereas objectives are collaboratively chosen by the engineering team.

${ }^{2}$ In this paper, we will use 'they' to refer to the decision body's multiple members, and 'it' to point to the decision body as a single or unitary collective. We also assume that multiple stakeholders in the decision body have multiple perspectives (e.g., different objectives toward the goal of a given assignment, or many preferences toward a single objective with multiple criteria). In other words, no dictatorship, unitary, or group-think among stakeholders in the collective will be considered.

${ }^{3}$ The world 'Value' in this paper refers to the Value-Focused Thinking framework, and should not be confused with the same word used in 'value function' in decision science (see Footnote next). In this paper, 'Value' is strictly used to refer to the highest level of human preferential system in group decisions (i.e., the 'Value-Perspective-Preference' hierarchy) as defined in Section 2.2.1.

${ }^{4}$ In decision science literatures, 'value function' is often used to represent human preference, such that the decision body's job is to choose an option that maximises this defined value function. In other literatures, the concept of 'utility function' is employed to quantify the 'true worth' (or utility) of the consequences of available alternatives; so that the best choose for the decision body is the alternative whose consequences lead to the highest 'expected utility'. Based on these definitions, it may seem that value refers preference and utility addresses alternative. However, in decision practice they are isomorphic and consistent with each other (with the exception that some may prefer to emphasise the fact that value is 'risk-neutral' and utility is 'risk-dependent' in decision making) - both providing means for the decision body to quantify preferences and compare alternatives. Nevertheless, the main difference lies within their representation details and manipulation procedures. To avoid the confusion, we do not use 'value function' for preferences and 'utility function' for alternatives in this paper. Instead, we use the term 'utility' to represent both utility function and value function that measures human preference, and reserve the word 'Value' for the 'high-level preference' in our Value $\rightarrow$ Perspective $\rightarrow$ Preference hierarchical structure (see Section 2.2.1) for group decisions. We then use 'ordinal utility' and 'cardinal utility' to represent the different levels of detail of measurability and comparability of human preference in our research (see Section 2.2.2).

${ }^{5}$ Note that from this point on in the paper, we will use the term 'decision body' to refer to a collective team which has multiple stakeholders that must make a Group decision $(G)$ jointly based on collective rationality established by themselves. We will refer to this decision body as 'they', instead of a 'he' or 'she', when pointing to its multiple stakeholders, and as 'it' when referring to this collective as a single Unitarian whole.

${ }^{6}$ As explained in Section 2.3.3, rationally and optimality are two different concepts and, therefore, this question should not be confused with the multi-objective optimisation concept. The best group decision based on collective rationality by multiple stakeholders in teamwork is not the same as the most optimised solution among the multiple objectives in task-work.

${ }^{7}$ Note that even when semi-transitivity requirement is justifiable (see Section 4.2 next), we still face the challenge of preference comparison and aggregation when attempting to reach collective rationality. Semi-transitivity (with the satisficing approach, see Section 2.3.2) weakens the exhaustiveness requirement of alternatives to be identified and compared; but it does not take away the need for interpersonal comparisons of individual preferences toward the remaining 'few promising alternatives'.

${ }^{8}$ This is the reason why traditional single-Objective optimisation methods can always produce 'rational' decisions for an individual. Optimality and rationality become conceptually isomorphic under single objective and single stakeholder ( $\mathrm{Lu}, 2007)$. 